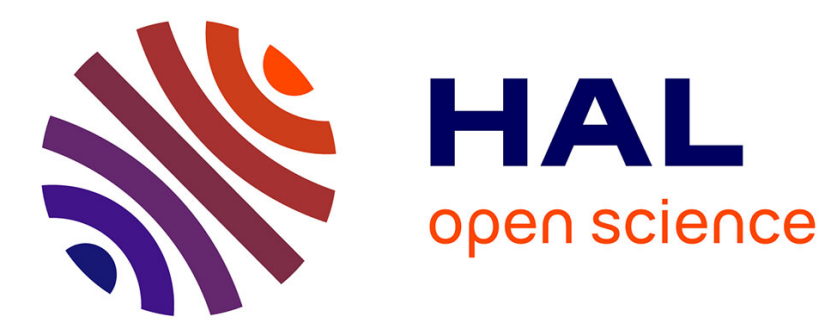

\title{
Daughter centrioles assemble preferentially towards the nuclear envelope in Drosophila syncytial embryos
}

\author{
Neil H J Cunningham, Imène B Bouhlel, Paul Conduit
}

\section{To cite this version:}

Neil H J Cunningham, Imène B Bouhlel, Paul Conduit. Daughter centrioles assemble preferentially towards the nuclear envelope in Drosophila syncytial embryos. Open Biology, 2022, 10.1098/rsob.210343 . hal-03454293v2

\section{HAL Id: hal-03454293 \\ https://hal.science/hal-03454293v2}

Submitted on 21 Jan 2022

HAL is a multi-disciplinary open access archive for the deposit and dissemination of scientific research documents, whether they are published or not. The documents may come from teaching and research institutions in France or abroad, or from public or private research centers.
L'archive ouverte pluridisciplinaire HAL, est destinée au dépôt et à la diffusion de documents scientifiques de niveau recherche, publiés ou non, émanant des établissements d'enseignement et de recherche français ou étrangers, des laboratoires publics ou privés. 
2 Daughter centrioles assemble preferentially towards the nuclear

7 Neil H. J. Cunningham ${ }^{1, \S}$, Imène B. Bouhlel ${ }^{1} \S$, and Paul T. Conduit ${ }^{1,2,3}$

8

$9 \quad{ }^{1}$ Department of Zoology, University of Cambridge, Downing Street, Cambridge, CB2 10 3EJ

11

12 2Université de Paris, CNRS, Institut Jacques Monod, F-75013, Paris, France.

13

$14 \S$ These authors contributed equally to this work.

$16{ }^{3}$ Corresponding author

17 Paul T. Conduit

18 Institut Jacques Monod

19 CNRS - Université de Paris

2015 rue Hélène Brion

2175013 Paris

22 France

$230033(1) 57278095$

24 paul.conduit@ijm.fr

25 ORCID: 0000-0002-7822-1191

26

27 Keywords: Centrosome, centriole, centriole duplication, Drosophila, microtubules. 


\section{Abstract}

29 Centrosomes are important organisers of microtubules within animal cells. They comprise a pair of centrioles surrounded by the pericentriolar material (PCM), which nucleates and organises the microtubules. To maintain centrosome numbers, centrioles must duplicate once and only once per cell cycle. During S-phase, a single new "daughter" centriole is built orthogonally on one side of each radially symmetric "mother" centriole. Mis-regulation of duplication can result in the simultaneous formation of multiple daughter centrioles around a single mother centriole, leading to centrosome amplification, a hallmark of cancer. It remains unclear how a single duplication site is established. It also remains unknown whether this site is pre-defined or randomly positioned around the mother centriole. Here, we show that within Drosophila syncytial embryos daughter centrioles preferentially assemble on the side of the mother facing the nuclear envelope, to which the centrosomes are closely attached. This positional preference is established early during duplication and remains stable throughout daughter centriole assembly, but is lost in centrosomes

43 forced to lose their connection to the nuclear envelope. This shows that noncentrosomal cues influence centriole duplication and raises the possibility that these external cues could help establish a single duplication site. 


\section{Introduction}

53 Centrosomes are important microtubule organising centres (MTOCs) within animal cells, best known for organising the mitotic spindle poles during cell division (Conduit et al., 2015b). They typically comprise an older "mother" and younger "daughter" pair of barrel-shaped microtubule-based centrioles. While centriole structure varies between species and cell type (Loncarek and Bettencourt-Dias, 2018), they all display a 9-fold radial symmetry, with an inner "cartwheel" structure supporting the assembly of 9 microtubule triplets, doublets or singlets that make up the centriole wall. The mother centriole recruits and organises a surrounding pericentriolar material (PCM), which contains the necessary microtubule-associating and signalling proteins required for centrosome function (Woodruff et al., 2014). The mother centriole also templates the assembly of the daughter centriole in a process called centriole duplication (Banterle and Gönczy, 2017; Firat-Karalar and Stearns, 2014; Fu et al., 2015). This occurs after cell division, when each daughter inherits a single centrosome containing a disengaged mother-daughter centriole pair. The daughter centriole is converted into a mother and both mothers support the orthogonal assembly of a new daughter centriole at their proximal end during S-phase. The two mother-daughter centriole pairs break apart during G2/M-phase to form two centrosomes, which mature by recruiting more PCM in preparation for mitosis. During mitosis, the two centrosomes each organise one pole of the bipolar spindle and towards the end of mitosis the centrioles disengage in preparation for a new round of duplication in the following cell cycle.

In most cell types, centrioles duplicate once per cell cycle during S-phase and it is this "once and only once" duplication event that maintains centrosome numbers through multiple cell divisions (Nigg and Holland, 2018). Failure to duplicate the centrioles during S-phase results in the inherence of a centrosome with a single centriole, which cannot then split to form two centrosomes. This leads to monopolar spindle formation and cell cycle arrest. In contrast, multiple centrosomes form if mother centrioles template the assembly of more than one daughter centriole and this leads to multipolar spindle formation in the next cell cycle. Multipolar spindles can result in cell death or 
attachments, leading to lagging chromosomes and chromosome instability (Basto et al., 2008; Ganem et al., 2009; Godinho and Pellman, 2014; Nigg and Holland, 2018). Centrosome amplification is strongly associated with cancer progression, with chromosome instability and increased centrosome signalling being possible causal links (Anderhub et al., 2012; Basto et al., 2008; Denu et al., 2016; Godinho et al., 2014; Godinho and Pellman, 2014; Mittal et al., 2021; Salisbury et al., 2004).

Seminal studies in $C$. elegans identified a core set of proteins necessary for centriole duplication: the kinase ZYG-1 and the large coiled-coil proteins SPD-2, SAS-4, SAS5 and SAS-6 (Delattre et al., 2006; Leidel and Gönczy, 2003; O'Connell et al., 2001; Pelletier et al., 2006). Homologues in Drosophila (Sak/Plk4, Spd-2, Sas-4, Ana2 and Sas-6) and human cells (PLK4, CEP192, CPAP, STIL and SAS-6) were subsequently identified and, with the exception of Drosophila Spd-2 (Dix and Raff, 2007), shown to also be essential for centriole duplication (Basto et al., 2006; Bettencourt-Dias et al., 2005; Dammermann et al., 2004; Habedanck et al., 2005; Kim et al., 2013; Leidel et al., 2005; Sonnen et al., 2013; Stevens et al., 2010; Tang et al., 2011; Terra et al., 2005). The role of worm SPD-2, which is to recruit ZYG1/PLK4, is played instead by Drosophila Asterless (Asl) (Blachon et al., 2008; Dzhindzhev et al., 2010), and the human homologues of Asl (CEP152) is also required for centriole duplication (Blachon et al., 2008; Cizmecioglu et al., 2010; Hatch et al., 2010), functioning together with the human homologue of SPD-2 (CEP192) to recruit PLK-4 (Kim et al., 2013; Park et al., 2014; Sonnen et al., 2013).

A large number of studies are producing a clear picture about how each of these proteins contributes to centriole assembly (reviewed in (Arquint and Nigg, 2016; FiratKaralar and Stearns, 2014; Fu et al., 2015; Yamamoto and Kitagawa, 2021). In essence, CEP192/SPD-2 and/or CEP152/AsI recruit the master kinase PLK-4 to the wall of the mother centriole where it regulates the recruitment of STIL/Ana2 and SAS6 and then CPAP/Sas-4 to form the daughter centriole. A key feature is that daughter centriole assembly occurs on only one side of the radially symmetric mother centriole, and this relies on localising PLK4, SAS-6 and STIL/Ana2 to a single spot on the side of the mother. The problem is that CEP192/SPD-2 and CEP152/Asl localise as a ring 
around the mother centriole and thus PLK4 is also initially recruited in a ring-like pattern (Kim et al., 2013; Park et al., 2014; Sonnen et al., 2013). In order for just a single daughter centriole to form, this ring of PLK4 must therefore be converted to a 'dot', which marks the site of centriole duplication. Failure of PLK4 to undergo this 'ring-to-dot' conversion results in multiple daughter centrioles forming around the mother centriole and this leads to centrosome amplification (Brownlee et al., 2011; Habedanck et al., 2005; Klebba et al., 2013; Kleylein-Sohn et al., 2007; Ohta et al., 2014). Ring-to-dot conversion of PLK4 is thought to be largely self-controlled, as it involves the auto-phosphorylation of a degron within PLK4 (Cunha-Ferreira et al., 2013, 2009; Guderian et al., 2010; Holland et al., 2010; Klebba et al., 2013; Rogers et al., 2009; Sillibourne et al., 2010), and could also depend on the ability of PLK4 to selfassemble, a property that is regulated by auto-phosphorylation and that protects PLK4 from degradation (Gouveia et al., 2018; Park et al., 2019; Yamamoto and Kitagawa, 2019). Nevertheless, ring-to-dot conversion is likely also influenced by the binding of STIL/Ana-2, which increases PLK4 activity (Arquint et al., 2015; Moyer et al., 2015) and protects PLK4 from degradation (Arquint et al., 2015; Ohta et al., 2014). In human cells, PLK4 is observed as an asymmetric punctate ring prior to the recruitment of STIL, suggesting that initial symmetry breaking is independent of STIL, although the full ring-to-dot conversion occurs only once STIL and SAS-6 have been recruited (Kim et al., 2013; Ohta et al., 2018, 2014; Park et al., 2014; Yamamoto and Kitagawa, 2019). In flies, Ana2 recruitment is the first observed symmetry breaking event (Dzhindzhev et al., 2017). Mathematical models can explain how the properties of PLK4, with or without the help of STIL/Ana2, can lead to the symmetry breaking ringto-dot transition (Leda et al., 2018; Takao et al., 2019).

While various studies have focussed on understanding how symmetry breaking is achieved, it remains unknown whether the site of daughter centriole assembly is randomly assigned or not. We decided to investigate this using Drosophila syncytial embryos as a model system. These embryos go through rapid and near-synchronous rounds of S-phase and then mitosis with no intervening gap phases. The nuclear envelope does not fully break down during mitosis and the centrosomes remain closely attached to the nuclear envelope throughout each cycle. At the end of mitosis / start 
148 of S-phase, mother and daughter centrioles separate with the daughter converting to

149 a mother and both centrioles quickly migrate around the nuclear envelope to form two

150 new centrosomes that will organise the next round of mitosis. During S-phase, each

151 mother centriole templates the formation of a new daughter centriole, with only the

152 mother centriole organising PCM (Conduit et al., 2015a, 2010). Towards the end of

153 mitosis, the centrioles disengage and the daughter centrioles are converted to mothers

154 by the addition of Asl, allowing them to begin recruiting PCM and initiate centriole

155 duplication in the next cycle (Conduit et al., 2010; Novak et al., 2014).

156

157 Using a dual-colour FRAP approach along with super-resolution Airyscan imaging, we show here that daughter centrioles preferentially assemble on the side of the mother centriole facing the nuclear envelope. By tracking duplication events throughout Sphase, we show that this preferential positioning of the daughter centriole with respect to the nucleus occurs from the early stages of centriole formation and remains relatively stable throughout the cycle. Using a point mutation in the key PCM protein

163 Centrosomin (Cnn), we show that this preferential positioning towards the nuclear 164 envelope is lost in centrosomes that have detached from the nuclear envelope. 165 Collectively, these observations suggest that the site of centriole duplication is influenced by the nuclear envelope and raise the possibility that cues external to the centriole duplication machinery may influence and help control centriole duplication. 
171 The site of daughter centriole assembly is non-random with respect to cell geometry

172 To address whether the site of daughter centriole formation is pre-defined or randomly 173 assigned during centriole duplication, we turned to the Drosophila syncytial embryo.

174 In these embryos hundreds of nuclei and centrosomes undergo rapid cycles of division

175 ( 8-15 min per cycle) in near synchrony, alternating between S-phase and M-phase

176 without gap phases. At around division cycle 9 the nuclei and centrosomes migrate to

177 the cell cortex and their divisions can be readily imaged with a fluorescence-based 178 microscope until they pause in cycle 14. Mitotic spindles form parallel to the cortex 179 such that they align along the $X-Y$ imaging plane. The mother centrioles also have a 180 regular alignment; their proximal-distal (end-to-end) axis is aligned orthogonally to the spindle axis such that mother centrioles point along the $Z$ imaging axis. Newly forming daughter centrioles grow along the $X-Y$ imaging axis. This regular alignment of the centrioles in theory allows one to record the position of the daughter centriole relative to other cellular structures, such as the mitotic spindle axis. Drosophila centrioles are relatively small, however, meaning that duplicating mother-daughter centriole pairs cannot be resolved using "standard" confocal microscopy. We therefore developed a method to estimate the location of the centrioles within an engaged mother-daughter centriole pair by performing dual-colour Fluorescence Recovery After Photobleaching (FRAP) experiments. This relies on the fact that PCM proteins, such as Spd-2, Asl or Cnn, are dynamically recruited around the mother, but not the daughter, centriole, while the centriole protein Sas-4 is dynamically recruited to the growing daughter, but not the mother, centriole (Conduit et al., 2015a). By tagging a PCM protein and Sas-4 with different coloured fluorophores and then photobleaching during S-phase, the centroids of the recovering fluorescent signals can be used to estimate the relative positions of the mother (PCM signal) and daughter centrioles (Sas-4 signal) (Figure 1A). We used this approach to compare the position of the growing daughter centriole relative to the mother centriole and the future spindle axis (Figure 1B).

To begin with, we used Spd-2-GFP and Sas-4-mCherry as our mother and daughter 200 centriole markers, respectively. We photobleached either one centrosome from a 
201 separating centrosome pair during early S-phase (when Sas-4 starts to be 202 incorporated at the newly forming daughter centriole) or we photobleached a single 203 centrosome in late M-phase, just prior to centrosome splitting, daughter centriole 204 assembly and Sas-4 recruitment, and monitored the two resulting centrosomes in the 205 following S-phase. Both cases result in centrosomes where Spd-2-GFP recovers only around the mother centriole and Sas-4-mCherry recovers only at the growing daughter centrioles during S-phase, but the latter case generates two centrosomes that can be analysed. We recorded the centroids of the recovering fluorescent signals in mid to late S-phase once the centrosomes had reached their final positions on the opposite side of the nuclear envelope. Waiting until the centrosomes had fully separated allowed us to use the future spindle axis (a line drawn between the paired centrosomes) as a spatial reference point with which to compare the position of daughter centriole assembly (Figure 1B). We analysed a total of 121 centrosomes from 16 embryos and collated the results. Strikingly, the positions of daughter centrioles were not evenly distributed relative to the future spindle axis (positive $\mathrm{Y}$ axis in Figure 1C). A frequency distribution of the angles of the daughter centrioles relative to the future spindle axis showed displayed a Normal distribution around the $0^{\circ}$ angle (Figure S1A,B) (passed all 4 normality tests in Prism) i.e. the daughter centrioles had a preference to be close to the $0^{\circ}$ angle and were not evenly distributed around the mother centriole (Chi-square $=44.52, \mathrm{df}=11, \mathrm{p}<0.0001$ ), as would be expected if daughter centriole positioning were random. The data can also be represented by a Rose Plot, where each segment corresponds to a duplication event and its position corresponds to the angle from the future spindle axis (Figure 1D). 95 of 121 (78.51\%) daughter centrioles were assembled within 90 degrees of the future spindle axis (blue segments, Figure 1D), while only 26 (21.49\%) were assembled more than 90 degrees from the future spindle axis (red segments, Figure 1F) (Binomial Wilson/Brown test, $\mathrm{p}<0.0001)$. The distribution of daughter centriole positions was not due to microscope induced misalignment of the green and red channels: auto-fluorescent beads were used to correct for microscope-induced offset between the channels (as in (Conduit et al., 2015a)); and the data was taken from multiple nuclei/centrosome pairs, all of which have different orientations with respect to the $X-Y$ axes of the microscope. Moreover, we observed a more random and non-Normal distribution of angles when imaging the 
fluorescence recovery of two PCM proteins, Spd-2-GFP and RFP-Cnn, which are expected to be closely aligned (Figure 1E,F; Figure S1C,D). Indeed, the positions of the recovering RFP-Cnn signals relative to the recovering Spd-2-GFP signals were much closer together with the mean distance between these signals $(0.099 \mu \mathrm{m})$ being significantly shorter than the mean distance between the recovering Spd-2-GFP (mother) and Sas-4-GFP (daughter) signals $(0.284 \mu \mathrm{m})$ (Figure $1 \mathrm{G})$. We also repeated the experiment using a green version of Sas-4 (Sas-4-GFP) and a different mother centriole marker (Asl-mCherry) on a different microscope and again found that the positions of daughter centriole assembly were not evenly distributed relative to the future spindle axis (Figure S1E), that the angles from the future spindle axis were Normally distributed around $0^{\circ}$ (Figure S1F,G), that a much higher proportion of daughter centrioles assembled within 90 degrees of the future spindle axis (Figure $\mathrm{S} 1 \mathrm{H})$, and that the distance between the recovering signals was similar to that for the Spd-2-GFP/Sas-4-mCherry data (Figure S1I). Collectively, this data shows that the positioning of daughter centriole assembly in Drosophila syncytial embryos is nonrandom with respect to cellular geometry.

The non-random position of daughter centriole assembly is dependent on centrosome association with the nuclear envelope

In Drosophila syncytial embryos, the centrosomes are tightly associated with the nuclear envelope via nuclear envelope associated Dynein (Robinson et al., 1999). Thus, the observation that daughter centrioles form preferentially within $90^{\circ}$ of the future spindle axis also meant that they were preferentially positioned on the side of the mother centriole facing the nuclear envelope. This raised the intriguing possibility that the nuclear envelope might influence the position of daughter centriole assembly. To test this, we wanted to examine the position of daughter centriole assembly in centrosomes that had detached from the nuclear envelope. We knew that Threonine 1133 within the PCM protein Cnn is important for Cnn to oligomerise and form a PCM scaffold (Feng et al., 2017) and our unpublished observations had shown that substituting Threonine 1133 with Alanine partially perturbs scaffold formation and the ability of centrosomes to remain attached to the nuclear envelope (see also Figure 
265 T1133A to analyse daughter centriole position in attached versus detached centrosomes. The detached centrosomes in Cnn-T1133A mutants normally remain

267

268

269

270

271

272

273

274

275

276

277

278

279

280

281

282

283

284

285

286

287

288

289

290

291

292

293

294

295

296 relatively close to the nuclear envelope, do not fall into the embryo centre, and form a spindle pole in during the following mitosis. Nevertheless, they often do not fully migrate around the nucleus (Figure 2A). Thus, instead of using the line between paired centrosomes as a reference point for the angle of daughter centriole assembly, we used a line drawn between the mother centriole and the centre of the nucleus (visualised due to the exclusion of fluorescence molecules), which we hereafter refer to as the nuclear axis (Figure $2 A, B$ ).

We photobleached centrosomes in late mitosis and monitored the fluorescence recovery during the following S-phase, noting which centrosomes had separated from the nuclear envelope and which had not. Importantly, the daughter centrioles within centrosomes that had remained attached to the nuclear envelope still displayed a preference to assemble on the side of the mother facing the nuclear envelope (Figure $2 \mathrm{C}-\mathrm{F}$ ), showing that perturbation of the PCM via Cnn's T1133A mutation did not indirectly affect daughter centriole positioning. In these attached centrosomes, the estimated position of the daughter centrioles displayed a similar non-even distribution to that observed in the analyses above for Spd-2-GFP;Sas-4-mCherry and AslmCherry; Sas-4-GFP (compare Figures 1C, 2C and Figure S1E). The measured angles of daughter centriole formation were normally distributed around $0^{\circ}$ (Figure 2D,E) (passed all 4 Normality tests in Prism) and a Rose Plot graph highlighted how $66.3 \%$ (59 of 89 ) daughter centrioles were positioned within 90 degrees of $0^{\circ}$ (Figure 2F) (Binomial Wilson/Brown test, $\mathrm{p}<0.01$ ). In contrast to the attached centrosomes, the daughter centrioles within centrosomes detached from the nuclear envelope did not display a preference to assemble on the side of the mother facing the nuclear envelope (Figure 2G-J). The estimated position of these daughter centrioles was more evenly spread around the mother centriole (Figure $2 \mathrm{G}$ ) and the angles at which they assembled relative to the nuclear axis were not normally distributed around $0^{\circ}$ (Figure $2 \mathrm{H}, \mathrm{I})$ (Failed 3 of 4 Normality tests in Prism) and were not significantly different from a random distribution (Chi-square $=8.4, \mathrm{df}=11, \mathrm{p}=0.68$ ). Moreover, there was no preference for the centrioles to form within 90 degrees of the nuclear axis, with similar 
numbers of daughter centrioles forming within 90 degrees (31/60) and more than 90 degrees (29/60) from the nuclear axis (Figure 2J) (Binomial Wilson/Brown test, $\mathrm{p}=0.90)$.

It was possible that the perceived loss of preference for the daughter centriole to form towards the nuclear axis in detached Cnn-T1133A centrosomes could have been an indirect effect of defects in centriole orientation with respect to the imaging axis i.e. detached centrosomes may tilt such that their daughter centrioles do not grow along the $X-Y$ imaging axis, causing increased noise and a possible randomising effect in the data. We ruled this out in two different ways. First, we compared the frequency at which GFP-Cnn-T1133A displayed a "central hole" at attached and detached centrosomes. Cnn molecules surround the mother centriole such that, with sufficient $X-Y$ spatial resolution, a "hole" in the centre of the Cnn fluorescence signal can be observed (e.g. top panels in Figure 3A, B). We reasoned that this central hole would be observed only in centrosomes that had their mother centriole pointing normally along the $Z$ imaging axis. We imaged fixed embryos in S-phase expressing GFP-CnnT1133A and Asl-mCherry (which labels only mother centrioles during S-phase) on a Zeiss Airyscan 2 microscope, which increases $X-Y$ spatial resolution to up to $120 \mathrm{~nm}$, and quantified the frequency of "clear", "partial", or "no clear" central holes in attached (Figure 3A) versus detached (Figure 3B) centrosomes. Out of a total of 112 centrosomes from 3 embryos, 83 were attached and 29 were detached. Of the 83 attached centrosomes, 38 (45.8\%) displayed a clear central hole, $25(30.1 \%)$ displayed a partial central hole, and $20(24.1 \%)$ displayed no clear central hole (Figure 3C). These percentages were similar in detached centrosomes. Of the 29 detached centrosomes, 12 (41.4\%) displayed a clear doughnut-like pattern, 9 (31.0\%) displayed a partial doughnut-like pattern, and 8 (27.6\%) displayed no clear doughnut-like pattern (Figure $3 \mathrm{C}$ ). There was no significant difference between the categorisation of these attached and detached centrosomes (Chi-square $=0.204$, $d f=2, p=0.903$ ), suggesting that detached centrosomes are not mis-oriented compared to attached centrosomes. To further support this finding, we used the previous Spd-2-GFP/Sas-4-mCherry FRAP data (Figure $2 \mathrm{C}, \mathrm{G}$ ) to compare the median estimated distances between mother and daughter centrioles in attached $(0.30 \mu \mathrm{m})$ versus detached $(0.33 \mu \mathrm{m})$ 
centrosomes and found there was no significant difference (Figure 3D; Mann-Whitney, $p=0.26$ ). The distance would in theory be shorter in detached centrosomes if they were misoriented. Thus, the data suggests that mother centrioles within centrosomes that have detached from the nuclear envelope remain aligned along the $Z$ imaging axis. We therefore conclude that, unlike in attached centrosomes, daughter centrioles within detached centrosomes do not form preferentially towards the nuclear envelope and that the nucleus somehow influences daughter centriole positioning.

\section{The positioning of daughter centriole assembly is consistent through time}

To estimate the position of daughter centrioles from our FRAP data, we had needed to wait until the fluorescent signals had recovered sufficiently in order to take accurate measurements, meaning that we could only assess daughter centriole positioning during mid to late S-phase. We therefore wondered whether the initial steps of daughter centriole formation occur with a positional preference, or whether they occur in a random position with the daughter centriole rotating to face the nuclear envelope later in S-phase. To address this, we performed live imaging of duplicating centrosomes throughout S-phase using an Airyscan microscope that enabled us to distinguish two mother and daughter foci of Sas-4-mCherry signal, with the mother centriole localised in the centre of the Spd-2-GFP fluorescence (Figure 4A). Note that the growing daughter centriole rapidly recruits excess Sas-4 (Conduit et al., 2015a) and so appears brighter than the mother for the majority of S-phase, and that Spd-2GFP, like GFP-Cnn, surrounds the mother centriole and can display a central hole with high enough spatial resolution (certain timepoints in Figure 4A; (Conduit et al., 2014)). Exclusion of cytoplasmic fluorescence can also be used to assess the position of the nuclear envelope (data not shown), which is indicated in blue in Figure 4A (note that centrosomes can migrate over the nucleus, explaining why the paired centrosome in timepoint 1 overlaps the nuclear region).

We followed 72 centrosomes for at least 6 timepoints ( $\sim 5$ minutes) and collated the data. Note that for most centrosomes, the mother and daughter centrioles within a pair were not resolvable for all 6 timepoints and so the number of measurements per timepoint varied between timepoints. We found that daughter centrioles had a strong 
361 preference to assemble on the side of the mother facing the nuclear envelope from

362 the earliest stage of S-phase that the daughter centrioles were visible (timepoint 1 ,

363 Figure 4B). Moreover, this preference remained throughout the 6 timepoints (Figure

364 4B). Indeed, we found that daughter centriole positioning relative to the nuclear axis

365 remained quite stable over time. The median angle deviation between timepoints was

$36621.5^{\circ}$, which is much lower than the median angle deviation expected were the

367 daughter centrioles to be positioned randomly at each timepoint $\left(\sim 90^{\circ}\right)$. Indeed, the

368 distribution of deviation angles was significantly different from the distribution of

369 random number data (Figure 4C; p<0.0001 Kolmogorov-Smirnov test). Collectively,

370 this data shows that daughter centriole assembly is initiated preferentially on the side

371 of the mother facing the nuclear envelope and that this positioning remains relatively

372 stable throughout daughter centriole assembly. 


\section{Discussion}

375 We have shown that during the mitotic nuclear cycles in Drosophila syncytial embryos daughter centrioles preferentially assemble on the side of the mother centriole facing the nuclear envelope. This preferential positioning is lost when centrosomes become detached from the nuclear envelope, raising the intriguing possibility that crosstalk between nuclear-envelope-related factors and the centriole duplication machinery may help to instruct centriole duplication. It remains to be seen whether an attachment to the nuclear envelope influences centriole duplication in other cell types, but the centrosome is not always attached to the nuclear envelope in all cell types. It remains possible, however, that other cellular structures could be involved in these cells, or that daughter centriole positioning is random, relying on stochastic processes.

We used a Cnn point mutant (Cnn-T1133A) to show that daughter centrioles within detached centrosomes lose their preferential positioning towards the nuclear envelope. This mutation has a relatively subtle effect on PCM assembly and stability, while being sufficient to cause a fraction of centrosomes to detach from the nuclear envelope. We predict that this detachment is stochastic and occurs because fewer microtubules can be organised at centrosomes with reduced PCM. Thus, there is a chance that some centrosomes will detach from the nuclear envelope. PCM levels are not low enough, however, to cause Cnn-T1133A centrosomes to 'rocket' around the embryo, unlike centrosomes completely lacking Cnn (Lucas and Raff, 2007), and we do not see any obvious effect on centriole structure or duplication. We therefore consider that the loss of preferential daughter centriole positioning in detached CnnT1133A centrosomes is not due to PCM instability per se, especially as the daughter centrioles within attached Cnn-T1133A centrosomes still retain a biased position towards the nuclear envelope. Although this bias (66\%) appears to be lower than that recorded when looking at "wild-type" centrosomes (78.5\% when using Spd-2-GFP and Sas-4-mCherry; 72.9\% when using Asl-mCherry and Sas-4-GFP), we believe this is due to a lower accuracy of estimating mother centriole position when using Cnn403 T1133A as opposed to using Spd-2 and Asl. This is because Spd-2 and Asl incorporate very close to the wall of the mother centriole, while Cnn incorporates into a broader area (Conduit et al., 2014), meaning that the recovering fluorescent signals 


\section{Opening Up}

420

421

422

423

424

of Spd-2 and Asl better predict mother centriole position. It would be satisfying to repeat the experiment with a mutation that perturbs centrosome attachment to the nuclear envelope without affecting centrosome structure at all, such as mutations in the LINC complex, but these experiments would be technically challenging due to the complex genetics and possible maternal effects of LINC mutants.

Further work is needed to understand the molecular basis for the positional bias, as well as understanding its importance, if any. We do not observe any obvious centriole duplication defects in Cnn-T1133A centrosomes that have detached from the nuclear envelope, indicating that the influence of the nuclear envelope on centriole duplication is not essential to ensure the production of a single daughter centriole, but we have not examined this extensively and it could still make the process more robust.

A major outstanding question is how PLK4 symmetry breaking is achieved to ensure that only one daughter centriole is formed on the side of the radially symmetric mother centriole (Yamamoto and Kitagawa, 2021). It is known that the PLK4 ring-to-dot transition requires proteasome activity (Ohta et al., 2014), Plk4 activity (Ohta et al., 2018; Park et al., 2019), and phosphorylation of PLK4's cryptic polo box (Park et al., 2019), suggesting that the auto-catalytic self-destructive properties of PLK4 could regulate the transition (Leda et al., 2018; Park et al., 2019; Takao et al., 2019; Yamamoto and Kitagawa, 2021, 2019). Indeed, computer modelling suggests that PLK4 symmetry breaking can be initiated by the self-organisational properties of PLK4 (Leda et al., 2018; Takao et al., 2019). An initial stochastic break in symmetry could then be enhanced by the binding of STIL (Leda et al., 2018; Takao et al., 2019), which both stimulates PLK4 activity (Moyer et al., 2015) and protects Plk4 from degradation (Arquint et al., 2015; Ohta et al., 2014). The different computer simulations place a difference emphasis on the role of STIL binding (Leda et al., 2018; Takao et al., 2019), but both agree that this is a critical step in completing the ring-to-dot transition. It is intriguing that STIL is able to bind to only a single site on the mother centriole even when PLK4 remains as a ring after proteasome inhibition (Ohta et al., 2014), suggesting that STIL recruitment to a single site within the ring of Plk4 could even be 
the initial trigger for symmetry breaking in certain circumstances. In Drosophila S2 cells, the first observed break in symmetry is the recruitment of the STIL homologue, Ana2, to a single spot on the mother centriole (Dzhindzhev et al., 2017).

Is there a link between PLK4, Ana2 and the nuclear envelope? In various cell types, including Drosophila syncytial embryos, the centrosomes are tightly associated with the nuclear envelope via interactions between the microtubules they organise and nuclear-envelope-associated Dynein (Agircan et al., 2014; Bolhy et al., 2011; Raaijmakers et al., 2012; Robinson et al., 1999; Splinter et al., 2010). From our observations, we speculate that molecules associated with the nuclear envelope or concentrated within the local environment between centrosomes and the nuclear envelope may help determine the position of centriole duplication proteins in Drosophila syncytial embryos. These putative molecules may help stabilise Plk4 or recruit Ana2, or both. This could relate to the asymmetry in centrosomal microtubules, with differences in the ability of the microtubules connecting the centrosomes to the nuclear envelope and the microtubules extending out into the cytosol to concentrate PLK4 and Ana2. Alternatively, perhaps proteins associated with the nuclear envelope can transiently bind Plk4 or Ana2 and thus increase their local concentration in the region between the mother centriole and nuclear envelope. Ana2 directly interacts with a conserved member of the Dynein complex, Cut-up (Ctp), which is a form of Dynein Light Chain in Drosophila (Slevin et al., 2014; Wang et al., 2011). Although the precise function of the Ana2-Ctp interaction remains unclear, it appears to help mediate Ana2 tetramerisation (Slevin et al., 2014), and Ana2 tetramerisation is important for centriole assembly (Cottee et al., 2015). Thus, while Ctp does not appear to be essential for centriole duplication (Wang et al., 2011), any Ctp molecules released from the nuclear associated Dynein complexes would be ideally positioned to bind to Ana2 and promote daughter centriole assembly on the side of the mother centriole facing the nuclear envelope.

It will also be interesting to see whether positional bias occurs in other systems. Intriguingly, LRRCC1 has recently been shown to localise asymmetrically within the lumen of human centrioles with the position of procentriole assembly being non- 
470 random with respect to this asymmetric mark (Gaudin et al., 2021). Thus, although the

471 molecular nature may vary, it's possible that a non-random positional preference in

472 daughter centriole assembly is an important conserved feature of centriole duplication.

473

474

475 Acknowledgements

476 This work was supported by a BBSRC New Investigator Award (BB/P019188/1), a

477 Wellcome Trust and Royal Society Sir Henry Dale Fellowship (105653/Z/14/Z) and an

478 IdEx Universite de Paris ANR-18-IDEX-0001 awarded to PTC. We thank Jordan Raff

479 for fly lines and the use of his spinning disk microscope. We thank Alan Wainman for

480 help with live Airyscan imaging. We thank Corinne Tovey for critical reading of the

481 manuscript. The work benefited from use of the imaging facility at the Stem Cell

482 Institute, University of Cambridge, the Micron imaging facility at the University of

483 Oxford, and the imaging facility at the Institut Jacques Monod, Université de Paris.

484 NHJC made the initial observation of positional preference by performing and 485 analysing dual FRAP experiments with Spd-2-GFP / Sas-4-mCherry and Spd-2-GFP 486 / RFP-Cnn, devised the formula to calculate angles, and performed and analysed the 487 live Airyscan experiments. IB collected additional data for the dual FRAP experiments 488 with Spd-2-GFP and Sas-4-mCherry and measured distances between centrioles in 489 detached versus attached centrioles. PTC designed the study, performed all other 490 experiments and analysis, and wrote the manuscript. The authors declare no financial 491 or non-financial competing interests.

492 
Figure 1

Analysis of dual-colour FRAP data reveals that the site of daughter centriole assembly is non-random. (A) Confocal images show a centrosome within an embryo expressing Spd-2-GFP (green) and Sas-4-mCherry (magenta) prior to photobleaching (left), immediately after photobleaching (centre), and after fluorescence recovery (right). The diagrams below are cartoon representations of how the proteins behave before and after photobleaching. Note that the recovering Sas-4-mCherry signal (daughter centriole) is offset from the centre of the recovering Spd-2-GFP signal

(B) Confocal image shows a pair of centrosomes (top unbleached, bottom recovering from bleaching) on opposite sides of the nuclear envelope (mid-late S-phase). The nuclear envelope and how angles from the future spindle axis are calculated are indicated.

(C) Graph displays the estimated positions of daughter centrioles (magenta circles) relative to the estimated position of their respective mother centrioles (position 0,0 on the graph) and the future spindle axis (positive yaxis) obtained from Spd-2-GFP (mother) Sas-4-mCherry (daughter) data. (D) Rose plot representing the angle at which daughter centrioles (marked by Sas-4-mCherry) form in relation to the future spindle axis $\left(0^{\circ}\right)$. Each segment corresponds to a single duplication event. Blue and red segments indicate daughter centriole assembly occurring less than or more than $90^{\circ}$ from the future spindle axis, respectively. (E)

514 Graph displays the positions of the centre of recovering RFP-Cnn signal relative to recovering Spd-2-GFP signal (position 0,0 on the graph) and the future spindle axis (positive y-axis) obtained from the control Spd-2-GFP (mother) RFP-Cnn (mother) data. (F) Rose plot (as in (D)) representing the angle relative to the future spindle axis $\left(0^{\circ}\right)$ formed by a line running between the recovering Spd-2-GFP and RFP-Cnn signals. (G) Graph showing the distance between the centre of the recovering Spd-2GFP signal (mother centriole) and the recovering Sas-4-mCherry signal (daughter centriole, magenta) or the recovering RFP-Cnn signal (mother centriole). The datasets were compared using a Mann-Whitney test.

\section{Figure 2}


525 The site of daughter centriole assembly is random in centrosomes that have

526 detached from the nuclear envelope. (A,B) Confocal image (A) and cartoon

527 representation $(B)$ show a pair of centrosomes in S-phase within an embryo

528 expressing GFP-Cnn-T1133A (grayscale). Note that one centrosome is attached to

529 and one centrosome is detached from the nuclear envelope. Cartoon in (B) indicates

530 how the angles of daughter centriole assembly from the nuclear axis were measured.

531 (C-J) Graphs display results from analysing the estimated position of daughter 532 centrioles relative to the estimated position of their respective mother centrioles 533 (position 0,0 on the graph) and the nuclear axis (positive y-axis) in centrosomes that 534 have either remained attached to $(C-F)$ or that have detached from (D-J) the nuclear 535 envelope within embryos expressing GFP-Cnn-T1133A and Sas-4-mCherry.

536 Estimated positions of the daughter centrioles were determined from analysing the 537 centre of fluorescence recovery of GFP-Cnn-T1133A (mother) and Sas-4-mCherry 538 (daughter). Graphs in $(C)$ and $(G)$ show the estimated positions of the daughter 539 centrioles; (D) and $(H)$ are frequency distributions of the angles at which daughter 540 centriole form in relation to the nuclear axis $\left(0^{\circ}\right)$; $(\mathrm{E})$ and $(\mathrm{I})$ are normal $\mathrm{QQ}$ plot showing 541 that the angles in (E), but not in (I), conform well to a Normal distribution; Rose plots 542 in $(F)$ and $(J)$ represent the angle at which daughter centrioles form in relation to the 543 mother centriole and the nuclear axis $\left(0^{\circ}\right)$. Each segment corresponds to a single 544 duplication event. Blue and red segments indicate daughter centriole assembly 545 occurring less than or more than $90^{\circ}$ from the nuclear axis, respectively.

546

547 Figure 3

548 Cnn-T1133A centrosomes that have detached from the nuclear envelope remain 549 correctly oriented with respect to the imaging axis. $(A, B)$ Airyscan images of 550 centrosomes that are either attached to $(A)$ or detached from $(B)$ the nuclear envelope 551 within embryos expressing GFP-Cnn-T1133A and Sas-4-mCherry in a cnn null mutant 552 background. Examples with a clear central hole (top panels), a partial central hole 553 (middle panels), and a no clear central hole (bottom panels) are shown. (C) Graph 554 shows the percentage of each centrosome type in either attached or detached 555 centrosomes, as indicated. Datasets were compared using a Chi-squared contingency 556 analysis. (D) Graph shows the distances between the estimated positions of mother 
557 and daughter centrioles from the Spd-2-GFP/Sas-4-mCherry FRAP data in either

558 attached or detached centrosomes, as indicated. The datasets were compared using

559 a Mann-Whitney test.

560

561 Figure 4

562 Daughter centrioles initially form preferentially towards the nuclear envelope

563 and retain a stable position throughout S-phase. (A) Airyscan images of a 564 centrosome in an embryo expressing Spd-2-GFP (green) and Sas-4-mCherry

565 (magenta) progressing through S-phase. Approximate times after centrosome splitting

566 are indicated - images were collected approximately every minute. The position of the

567 nuclear envelope (as determined by the exclusion of fluorescence from the nucleus)

568 is indicated by the dotted blue line. The Sas-4-mCherry signals for mother $(\mathrm{m})$ and

569 daughter (d) centrioles are also indicated. (B) Rose plot graphs display the angle at

570 which daughter centrioles form in relation to the mother centriole and the nuclear axis

$571\left(0^{\circ}\right)$ as calculated from time-lapse Airyscan images that followed centrosomes

572 throughout S-phase. Each segment corresponds to a single duplication event. Blue

573 and red segments indicate daughter centriole assembly occurring less than or more

574 than $90^{\circ}$ from the nuclear axis, respectively. Each rose plot corresponds to a given

575 timepoint, with timepoint 1 occurring $\sim 1$ minute after centrosome splitting and there

576 being a $\sim 1$-minute gap between timepoints. The numbers of events for each timepoint

577 are indicated; this varies due to the varying ability to resolve the two centrioles through

578 time. (C) Graph shows the change in the angle of the daughter centriole (angle

579 deviation) with respect to the mother centriole and the nuclear axis that occurred

580 between timepoints from real data (left dataset) or randomly generated angles (right

581 dataset). Each point on the graph represents an individual angle deviation. The

582 median and $95 \% \mathrm{Cls}$ are shown. The $\mathrm{p}$ value indicates that the two datasets have a

583 different distribution (Kolmogorov-Smirnov test). 


\section{Contact for Reagent and Resource Sharing}

587 Further information and requests for resources and reagents should be directed to and will be fulfilled by the Lead Contact, Paul Conduit (paul.conduit@ijm.fr).

\section{Experimental Model and Subject Details}

All fly strains were maintained at 18 or $25^{\circ} \mathrm{C}$ on Iberian fly food made from dry active yeast, agar, and organic pasta flour, supplemented with nipagin, propionic acid, pen/strep and food colouring.

\section{Methods}

596 Drosophila melanogaster stocks

597 The following fluorescent alleles were used in this study: pUbq-Spd-2-GFP (Dix and Raff, 2007), eSas-4-mCherry (endogenous promoter) (Conduit et al., 2015a), pUbqRFP-Cnn (Conduit et al., 2010), eSas-4-GFP (endogenous promoter) (Novak et al., 2014), eAsI-mCherry (endogenous promoter) (Conduit et al., 2015a), pUbq-GFP-Cnn601 T1133A (this study). To make the pUbq-GFP-Cnn-T1133A allele, we used QuikChange (Agilent) to introduce the T1133A mutation into Cnn within a pDONR vector and used Gateway cloning (ThermoFisher) to transfer it into a pUbq-GFP vector containing a miniwhite marker. This construct was injected by BestGene in order to generate transgenic lines.

606

607 For performing FRAP experiments we used fly lines expressing either: two copies of pUbq-Spd-2-GFP and two copies eSas-4-mCherry in a sas-4 null background (sas4/(3)2214/Df(3R)BSC221); two copies of pUbq-Spd-2-GFP and one copy of RFP-Cnn in a cnn ${ }^{\text {f04547/ }} \mathrm{cnn}^{\mathrm{HK} 21}$ mutant background; two copies of eSas-4-GFP and two copies of eAsl-mCherry in a sas-4 null background (sas-4|(3)2214/Df(3R)BSC221); or one copy of pUbq-GFP-Cnn-T1133A and two copies eSas-4-mCherry in a sas-4 null background (sas-4/(3)2214/Df(3R)BSC221). For the live Airyscan imaging, we used flies expressing

614 two copies of pUbq-Spd-2-GFP and two copies eSas-4-mCherry in a sas-4 null background (sas-4/(3)2214/Df(3R)BSC221). For the fixed Airyscan imaging, we used 
flies expressing one copy of pUbq-GFP-Cnn-T1133A and two copies eAsl-mCherry in an asl null mutant background (asImecd (Blachon et al., 2008)).

\section{Fixed and live cell imaging}

620 For live dual FRAP experiments, $0.5 \mu \mathrm{m}$ thick confocal sections were collected from 621 living syncytial embryos in nuclear cycle 11 or 12 at $\sim 21^{\circ} \mathrm{C}$ on either a Perkin Elmer 622 ERS Spinning Disk confocal system mounted on a Zeiss Axiovert microscope using a 623 63X/1.4NA Oil objective, or an Andor Revolution Spinning Disk confocal system 624 mounted on a Nikon Ti inverted microscope coupled to an Andor iXon camera using 625 a Plan-Apochromat 60X/1.4NA Oil objective. Focused 488nm and $561 \mathrm{~nm}$ lasers were 626 used to photobleach the GFP and mCherry/RFP signals, respectively. For live 627 Airyscan imaging, $0.2 \mu \mathrm{m}$ thick sections were collected from living embryos in nuclear 628 cycle 12 or 13 on an inverted Zeiss 880 microscope fitted with an Airyscan detector 629 at $21^{\circ} \mathrm{C}$ and a Plan-Apochromat $63 \times / 1.4 \mathrm{NA}$ oil lens using 488-nm argon and 561-nm 630 diode lasers. Images were collected approximately every $1 \mathrm{~min}$ with a zoom value of 63123.3 pixels/ $\mu \mathrm{m}$. Focus was readjusted between the 1-min intervals. Images were Airy632 processed in 3D with a strength value of "auto" ( 6) or 6.5. For fixed Airyscan imaging, $6330.2 \mu \mathrm{m}$ thick sections were collected from methanol fixed embryos in nuclear cycle 11 634 or 12 on an inverted Zeiss LSM980 microscope fitted with an Airyscan2 detector at $21^{\circ} \mathrm{C}$ and a Plan-Apochromat $63 \times / 1.4$ NA oil lens using 488-nm argon and 561-nm diode lasers. When measuring centriole positions, images from the different colour channels were registered with alignment parameters obtained from calibration measurements with $0.2 \mu \mathrm{m}$ diameter TetraSpeck beads (Life Technologies). The centroids of each fluorescent signal were calculated in ImageJ using the "centre of mass" analysis tool. The number of pixels for the images was first increased such that each real pixel was made of $5 \times 5$ sub-pixels. This increases the location accuracy for the centroid of the fluorescence signal.

\section{Quantification and Statistical Analysis}

645 Data was processed in Microsoft Excel. Graph production was performed using either 646 Microsoft Excel (rose plots) or GraphPad Prism (all other graphs) and statistical 647 analysis was performed using GraphPad Prism. N numbers and statistical tests used 
648 for each experiment are indicated within the main text or Figure Legends. The 649 following Normality tests were carried out in Prism to analyse the frequency 650 distributions of angles: Anderson-Darling test, D’Agostino \& Pearson test, Shapiro651 Wilk test, Kolmogorov-Smirnov test.

652

653

654 
Agircan FG, Schiebel E, Mardin BR. 2014. Separate to operate: control of centrosome positioning and separation. Philosophical Transactions Royal Soc $B$ Biological Sci 369:20130461 20130461. doi:10.1098/rstb.2013.0461

Anderhub SJ, Krämer A, Maier B. 2012. Centrosome amplification in tumorigenesis.

Arquint C, Gabryjonczyk A-M, Imseng S, Böhm R, Sauer E, Hiller S, Nigg EA, Maier T. 2015. STIL binding to Polo-box 3 of PLK4 regulates centriole duplication. Elife 4:e07888. doi:10.7554/elife.07888

Arquint C, Nigg EA. 2016. The PLK4-STIL-SAS-6 module at the core of centriole duplication. Biochem Soc T 44:1253-1263. doi:10.1042/bst20160116

Banterle N, Gönczy P. 2017. Centriole Biogenesis: From Identifying the Characters to Understanding the Plot. Annu Rev Cell Dev Bi 33:23 49. doi:10.1146/annurevcellbio-100616-060454

Basto R, Brunk K, Vinadogrova T, Peel N, Franz A, Khodjakov A, Raff JW. 2008. Centrosome amplification can initiate tumorigenesis in flies. Cell 133:1032 1042. doi:10.1016/j.cell.2008.05.039 Flies without centrioles. Cell 125:1375 1386. doi:10.1016/j.cell.2006.05.025

Bettencourt-Dias M, Rodrigues-Martins A, Carpenter L, Riparbelli M, Lehmann L, Gatt MK, Carmo N, Balloux F, Callaini G, Glover DM. 2005. SAK/PLK4 is required for centriole duplication and flagella development. Curr Biol 15:2199 2207. doi:10.1016/j.cub.2005.11.042

Blachon S, Gopalakrishnan J, Omori Y, Polyanovsky A, Church A, Nicastro D, Malicki J, Avidor-Reiss T. 2008. Drosophila asterless and vertebrate Cep152 Are orthologs essential for centriole duplication. Genetics 180:2081 2094. doi:10.1534/genetics.108.095141

Bolhy S, Bouhlel I, Dultz E, Nayak T, Zuccolo M, Gatti X, Vallee R, Ellenberg J, Doye V. 2011. A Nup133-dependent NPC-anchored network tethers centrosomes to the nuclear envelope in prophase. J Cell Biology 192:855-871. doi:10.1083/jcb.201007118 Biology 195:231-243. doi:10.1083/jcb.201107086 
Cizmecioglu O, Arnold M, Bahtz R, Settele F, Ehret L, Haselmann-Weiß U, Antony C, Hoffmann I. 2010. Cep152 acts as a scaffold for recruitment of Plk4 and CPAP to the centrosome. J Cell Biology 191:731-739. doi:10.1083/jcb.201007107

Conduit PT, Brunk K, Dobbelaere J, Dix CI, Lucas EP, Raff JW. 2010. Centrioles regulate centrosome size by controlling the rate of $\mathrm{Cnn}$ incorporation into the PCM. Curr Biol 20:2178 2186. doi:10.1016/j.cub.2010.11.011

Conduit PT, Richens JH, Wainman A, Holder J, Vicente CC, Pratt MB, Dix CI, Novak ZA, Dobbie IM, Schermelleh L, Raff JW. 2014. A molecular mechanism of mitotic centrosome assembly in Drosophila. Elife 3:2987. doi:10.7554/elife.03399

Conduit PT, Wainman A, Novak ZA, Weil TT, Raff JW. 2015a. Re-examining the role of Drosophila Sas-4 in centrosome assembly using two-colour-3D-SIM FRAP. Elife 4:e08483. doi:10.7554/elife.08483

Conduit PT, Wainman A, Raff JW. 2015b. Centrosome function and assembly in animal cells. Nat Rev Mol Cell Bio 16:611 624. doi:10.1038/nrm4062

Cottee MA, Muschalik N, Johnson S, Leveson J, Raff JW, Lea SM. 2015. The homooligomerisation of both Sas- 6 and Ana2 is required for efficient centriole assembly in flies. Elife 4:e07236. doi:10.7554/elife.07236

Cunha-Ferreira I, Bento I, Pimenta-Marques A, Jana SC, Lince-Faria M, Duarte P, Borrego-Pinto J, Gilberto S, Amado T, Brito D, Rodrigues-Martins A, Debski J, Dzhindzhev N, Bettencourt-Dias M. 2013. Regulation of autophosphorylation controls PLK4 self-destruction and centriole number. Curr Biol 23:2245 2254. doi:10.1016/j.cub.2013.09.037

Cunha-Ferreira I, Rodrigues-Martins A, Bento I, Riparbelli M, Zhang W, Laue E, Callaini G, Glover DM, Bettencourt-Dias M. 2009. The SCF/Slimb ubiquitin ligase limits centrosome amplification through degradation of SAK/PLK4. Curr Biol 19:43 49. doi:10.1016/j.cub.2008.11.037

Dammermann A, Müller-Reichert T, Pelletier L, Habermann B, Desai A, Oegema K. 2004. Centriole Assembly Requires Both Centriolar and Pericentriolar Material Proteins. Dev Cell 7:815-829. doi:10.1016/j.devcel.2004.10.015

Delattre M, Canard C, Gönczy P. 2006. Sequential protein recruitment in C. elegans centriole formation. Curr Biol 16:1844 1849. doi:10.1016/j.cub.2006.07.059

Denu RA, Zasadil LM, Kanugh C, Laffin J, Weaver BA, Burkard ME. 2016. Centrosome amplification induces high grade features and is prognostic of worse outcomes in breast cancer. Bmc Cancer 16:47. doi:10.1186/s12885-016-2083-x

Dix CI, Raff JW. 2007. Drosophila Spd-2 Recruits PCM to the Sperm Centriole, but Is Dispensable for Centriole Duplication. Curr Biol 17:1759-1764. doi:10.1016/j.cub.2007.08.065 
Dzhindzhev NS, Tzolovsky G, Lipinszki Z, Abdelaziz M, Debski J, Dadlez M, Glover DM. 2017. Two-step phosphorylation of Ana2 by Plk4 is required for the sequential loading of Ana2 and Sas6 to initiate procentriole formation. Open Biol 7:170247. doi:10.1098/rsob.170247

Dzhindzhev NS, Yu QD, Weiskopf K, Tzolovsky G, Cunha-Ferreira I, Riparbelli M, Rodrigues-Martins A, Bettencourt-Dias M, Callaini G, Glover DM. 2010. Asterless is a scaffold for the onset of centriole assembly. Nature 467:714 718. doi:10.1038/nature09445

Feng Z, Caballe A, Wainman A, Johnson S, Haensele AFM, Cottee MA, Conduit PT, Lea SM, Raff JW. 2017. Structural Basis for Mitotic Centrosome Assembly in Flies. Cell 169:1078 1089.e13. doi:10.1016/j.cell.2017.05.030

Firat-Karalar EN, Stearns T. 2014. The centriole duplication cycle. Philosophical Transactions Royal Soc B Biological Sci 369:20130460-20130460. doi:10.1098/rstb.2013.0460

Fu J, Hagan IM, Glover DM. 2015. The Centrosome and Its Duplication Cycle. Csh Perspect Biol 7:a015800. doi:10.1101/cshperspect.a015800

Ganem N, Godinho S, Pellman D. 2009. A mechanism linking extra centrosomes to chromosomal instability. Nature 460. doi:10.1038/nature08136

Gaudin N, Gil PM, Boumendjel M, Ershov D, Pioche-Durieu C, Bouix M, Delobelle Q, Maniscalco L, Phan TBN, Heyer V, Reina-San-Martin B, Azimzadeh J. 2021. Evolutionary conservation of centriole rotational asymmetry in the human centrosome. Biorxiv 2021.07.21.453218. doi:10.1101/2021.07.21.453218

Godinho SA, Pellman D. 2014. Causes and consequences of centrosome abnormalities in cancer. Philosophical Transactions Royal Soc B Biological Sci 369:20130467-20130467. doi:10.1098/rstb.2013.0467

Godinho SA, Picone R, Burute M, Dagher R, Su Y, Leung CT, Polyak K, Brugge JS, Théry M, Pellman D. 2014. Oncogene-like induction of cellular invasion from centrosome amplification. Nature 510:167 171. doi:10.1038/nature13277

Gouveia SM, Zitouni S, Kong D, Duarte P, Gomes BF, Sousa AL, Tranfield EM, Hyman A, Loncarek J, Bettencourt-Dias M. 2018. PLK4 is a microtubuleassociated protein that self assembles promoting de novo MTOC formation. $J$ Cell Sci 132:jcs.219501. doi:10.1242/jcs.219501

Guderian G, Westendorf J, Uldschmid A, Nigg EA. 2010. Plk4 transautophosphorylation regulates centriole number by controlling betaTrCP-mediated degradation. J Cell Sci 123:2163 2169. doi:10.1242/jcs.068502

Habedanck R, Stierhof Y-D, Wilkinson CJ, Nigg EA. 2005. The Polo kinase Plk4 functions in centriole duplication. Nat Cell Biol 7:1140 1146. doi:10.1038/ncb1320 
Hatch EM, Kulukian A, Holland AJ, Cleveland DW, Stearns T. 2010. Cep152 interacts with Plk4 and is required for centriole duplication. J Cell Biology 191:721-729. doi:10.1083/jcb.201006049

Holland AJ, Lan W, Niessen S, Hoover H, Cleveland DW. 2010. Polo-like kinase 4 kinase activity limits centrosome overduplication by autoregulating its own stability. J Cell Biology 188:191-198. doi:10.1083/jcb.200911102

Kim T-S, Park J-E, Shukla A, Choi S, Murugan RN, Lee JH, Ahn M, Rhee K, Bang JK, Kim BY, Loncarek J, Erikson RL, Lee KS. 2013. Hierarchical recruitment of Plk4 and regulation of centriole biogenesis by two centrosomal scaffolds, Cep192 and Cep152. Proc National Acad Sci 110:E4849-E4857. doi:10.1073/pnas.1319656110

Klebba JE, Buster DW, Nguyen AL, Swatkoski S, Gucek M, Rusan NM, Rogers GC. 2013. Polo-like Kinase 4 Autodestructs by Generating Its Slimb-Binding Phosphodegron. Curr Biol 23:2255-2261. doi:10.1016/j.cub.2013.09.019

Kleylein-Sohn J, Westendorf J, Clech ML, Habedanck R, Stierhof Y-D, Nigg EA. 2007. Plk4-induced centriole biogenesis in human cells. Dev Cell 13:190 202. doi:10.1016/j.devcel.2007.07.002

Leda M, Holland AJ, Goryachev AB. 2018. Autoamplification and competition drive symmetry breaking: Initiation of centriole duplication by the PLK4-STIL network. Iscience 8:222 235. doi:10.1016/j.isci.2018.10.003

Leidel S, Delattre M, Cerutti L, Baumer K, Gönczy P. 2005. SAS-6 defines a protein family required for centrosome duplication in $\mathrm{C}$. elegans and in human cells. Nat Cell Biol 7:115-125. doi:10.1038/ncb1220

Leidel S, Gönczy P. 2003. SAS-4 Is Essential for Centrosome Duplication in C. elegans and Is Recruited to Daughter Centrioles Once per Cell Cycle. Dev Cell 4:431-439. doi:10.1016/s1534-5807(03)00062-5

Loncarek J, Bettencourt-Dias M. 2018. Building the right centriole for each cell type. J Cell Biol 217:823-835. doi:10.1083/jcb.201704093

Lucas EP, Raff JW. 2007. Maintaining the proper connection between the centrioles and the pericentriolar matrix requires Drosophila Centrosomin. J Cell Biology 178:725-732. doi:10.1083/jcb.200704081

Mittal K, Kaur J, Jaczko M, Wei G, Toss MS, Rakha EA, Janssen EAM, Søiland H, Kucuk O, Reid MD, Gupta MV, Aneja R. 2021. Centrosome amplification: a quantifiable cancer cell trait with prognostic value in solid malignancies. Cancer Metast Rev 40:319-339. doi:10.1007/s10555-020-09937-z 
Moyer TC, Clutario KM, Lambrus BG, Daggubati V, Holland AJ. 2015. Binding of STIL to Plk4 activates kinase activity to promote centriole assembly. J Cell Biology 209:863-878. doi:10.1083/jcb.201502088

Nigg EA, Holland AJ. 2018. Once and only once: mechanisms of centriole duplication and their deregulation in disease. Nat Rev Mol Cell Bio 19:297-312. doi:10.1038/nrm.2017.127

Novak ZA, Conduit PT, Wainman A, Raff JW. 2014. Asterless licenses daughter centrioles to duplicate for the first time in Drosophila embryos. Curr Biol 24:1276 1282. doi:10.1016/j.cub.2014.04.023

O'Connell KF, Caron C, Kopish KR, Hurd DD, Kemphues KJ, Li Y, White JG. 2001. The C. elegans zyg-1 Gene Encodes a Regulator of Centrosome Duplication with Distinct Maternal and Paternal Roles in the Embryo. Cell 105:547-558. doi:10.1016/s0092-8674(01)00338-5

Ohta M, Ashikawa T, Nozaki Y, Kozuka-Hata H, Goto H, Inagaki M, Oyama M, Kitagawa D. 2014. Direct interaction of Plk4 with STIL ensures formation of a single procentriole per parental centriole. Nat Commun 5:5267. doi:10.1038/ncomms6267

Ohta M, Watanabe K, Ashikawa T, Nozaki Y, Yoshiba S, Kimura A, Kitagawa D. 2018. Bimodal Binding of STIL to Plk4 Controls Proper Centriole Copy Number. Cell Reports 23:3160-3169.e4. doi:10.1016/j.celrep.2018.05.030

Park J-E, Zhang L, Bang JK, Andresson T, DiMaio F, Lee KS. 2019. Phase separation of Polo-like kinase 4 by autoactivation and clustering drives centriole biogenesis. Nat Commun 10:4959. doi:10.1038/s41467-019-12619-2

Park S-Y, Park J-E, Kim T-S, Kim JH, Kwak M-J, Ku B, Tian L, Murugan RN, Ahn M, Komiya S, Hojo H, Kim N-H, Kim BY, Bang JK, Erikson RL, Lee KW, Kim SJ, Oh B-H, Yang W, Lee KS. 2014. Molecular Basis for Unidirectional Scaffold Switching of Human Plk4 in Centriole Biogenesis. Nat Struct Mol Biol 21:696-703. doi:10.1038/nsmb.2846

Pelletier L, O'Toole E, Schwager A, Hyman AA, Müller-Reichert T. 2006. Centriole assembly in Caenorhabditis elegans. Nature 444:619 623. doi:10.1038/nature05318

Raaijmakers JA, Heesbeen RGHP van, Meaders JL, Geers EF, Fernandez-Garcia B, Medema RH, Tanenbaum ME. 2012. Nuclear envelope-associated dynein drives prophase centrosome separation and enables Eg5-independent bipolar spindle formation. Embo J 31:4179 4190. doi:10.1038/emboj.2012.272

Robinson JT, Wojcik EJ, Sanders MA, McGrail M, Hays TS. 1999. Cytoplasmic dynein is required for the nuclear attachment and migration of centrosomes during mitosis in Drosophila. The Journal of cell biology 146:597 608. 
Rogers GC, Rusan NM, Roberts DM, Peifer M, Rogers SL. 2009. The SCFSlimb ubiquitin ligase regulates Plk4/Sak levels to block centriole reduplication. J Cell Biology 184:225-239. doi:10.1083/jcb.200808049

Salisbury JL, D’Assoro AB, Lingle WL. 2004. Centrosome Amplification and the Origin of Chromosomal Instability in Breast Cancer. J Mammary Gland Biol 9:275-283. doi:10.1023/b:jomg.0000048774.27697.30

Sillibourne JE, Tack F, Vloemans N, Boeckx A, Thambirajah S, Bonnet P, Ramaekers FCS, Bornens M, Grand-Perret T. 2010. Autophosphorylation of pololike kinase 4 and its role in centriole duplication. Mol Biol Cell 21:547 561. doi:10.1091/mbc.e09-06-0505

Slevin LK, Romes EM, Dandulakis MG, Slep KC. 2014. The Mechanism of Dynein Light Chain LC8-mediated Oligomerization of the Ana2 Centriole Duplication Factor. J Biol Chem 289:20727-20739. doi:10.1074/jbc.m114.576041

Sonnen KF, Gabryjonczyk A-M, Anselm E, Stierhof Y-D, Nigg EA. 2013. Human Cep192 and Cep152 cooperate in Plk4 recruitment and centriole duplication. $J$ Cell Sci 126:3223 3233. doi:10.1242/jcs.129502

Splinter D, Tanenbaum ME, Lindqvist A, Jaarsma D, Flotho A, Yu KL, Grigoriev I, Engelsma D, Haasdijk ED, Keijzer N, Demmers J, Fornerod M, Melchior F, Hoogenraad CC, Medema RH, Akhmanova A. 2010. Bicaudal D2, dynein, and kinesin-1 associate with nuclear pore complexes and regulate centrosome and nuclear positioning during mitotic entry. Plos Biol 8:e1000350. doi:10.1371/journal.pbio.1000350

Stevens NR, Dobbelaere J, Brunk K, Franz A, Raff JW. 2010. Drosophila Ana2 is a conserved centriole duplication factor. J Cell Biology 188:313-323. doi:10.1083/jcb.200910016

Takao D, Yamamoto S, Kitagawa D. 2019. A theory of centriole duplication based on self-organized spatial pattern formation. J Cell Biol 218:3537-3547. doi:10.1083/jcb.201904156

Tang CC, Lin S, Hsu W, Lin Yi-Nan, Wu C, Lin Yu-Chih, Chang C, Wu K, Tang TK. 2011. The human microcephaly protein STIL interacts with CPAP and is required for procentriole formation. Embo J 30:4790-4804. doi:10.1038/emboj.2011.378

Terra SL, English CN, Hergert P, McEwen BF, Sluder G, Khodjakov A. 2005. The de novo centriole assembly pathway in HeLa cells cell cycle progression and centriole assembly/maturation. J Cell Biology 168:713-722. doi:10.1083/jcb.200411126

Wang C, Li S, Januschke J, Rossi F, Izumi Y, Garcia-Alvarez G, Gwee SSL, Soon SB, Sidhu HK, Yu F, Matsuzaki F, Gonzalez C, Wang H. 2011. An ana2/ctp/mud 
875 complex regulates spindle orientation in Drosophila neuroblasts. Dev Cell 21:520

876 533. doi:10.1016/j.devcel.2011.08.002

877 Woodruff JB, Wueseke O, Hyman AA. 2014. Pericentriolar material structure and 878 dynamics. Philosophical Transactions Royal Soc B Biological Sci 369:20130459.

879 doi:10.1098/rstb.2013.0459

880 Yamamoto S, Kitagawa D. 2021. Emerging insights into symmetry breaking in 881 centriole duplication: updated view on centriole duplication theory. Curr Opin 882 Struc Biol 66:8-14. doi:10.1016/j.sbi.2020.08.005

883 Yamamoto S, Kitagawa D. 2019. Self-organization of Plk4 regulates symmetry breaking in centriole duplication. Nat Commun 10:1810. doi:10.1038/s41467-01909847-x 


\section{Figure 1}

A

mother/daughter centrioles cannot be distinguished
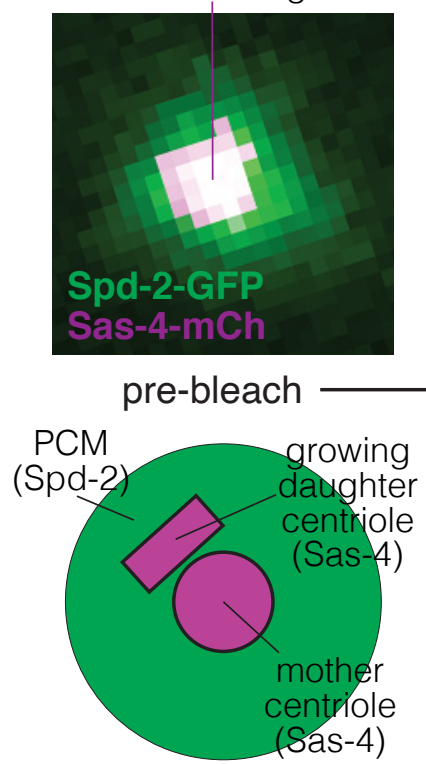

centre of mass of recovering signal estimated position of estimated position of daughter centriole mother centriole

B
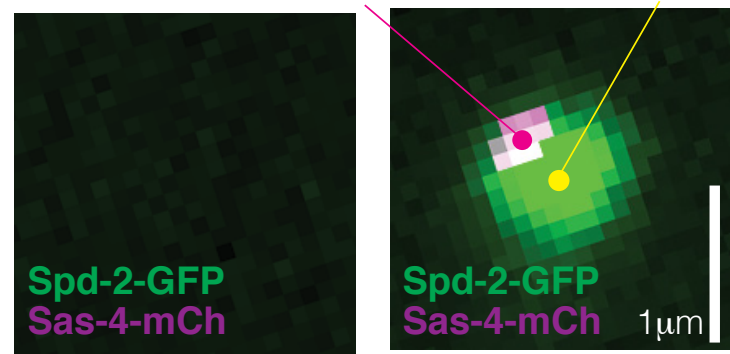

photobleach $\rightarrow$ fluorescence recovery
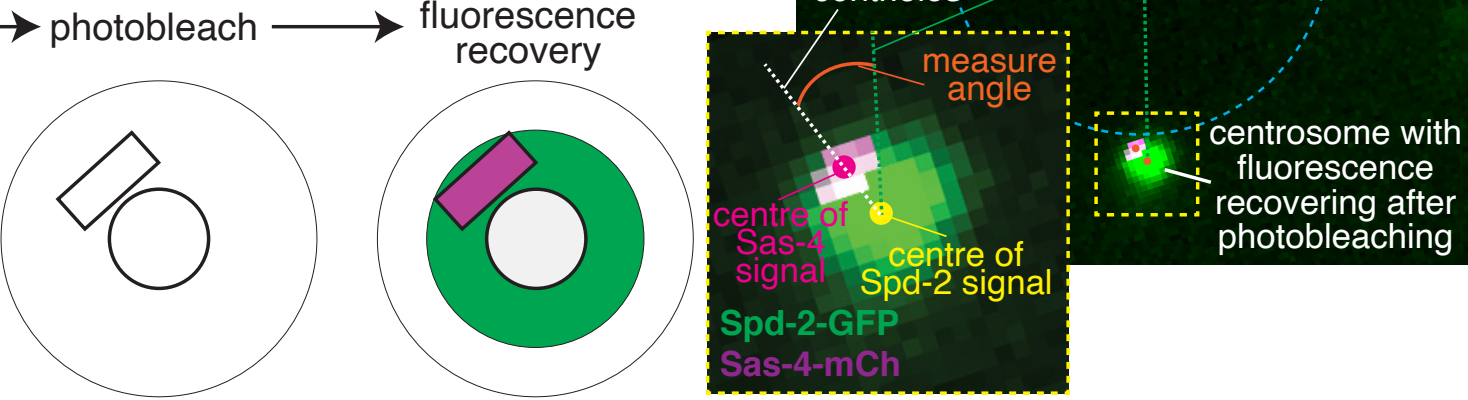

\section{mother-daughter experiment}

\section{C daughter centriole position}

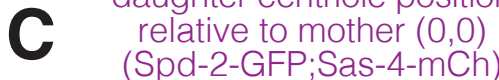

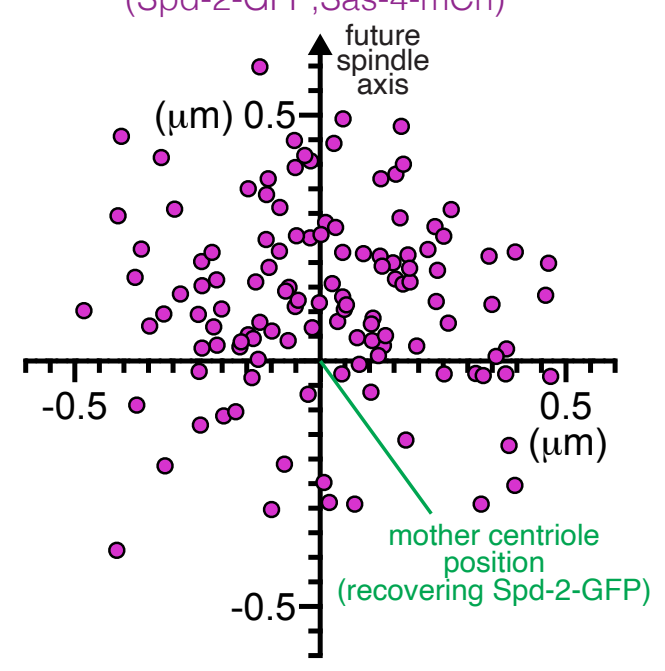

\section{mother-mother control}

E

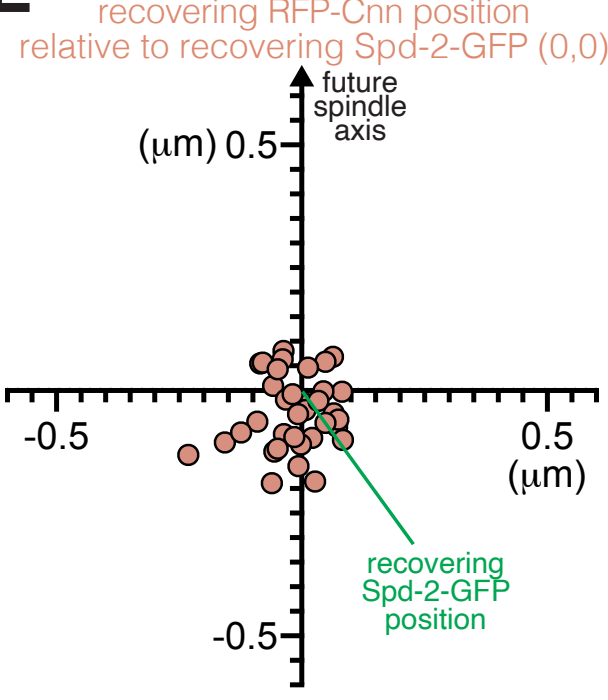

D rose plot for daughter centriole angle relative to future spindle axis

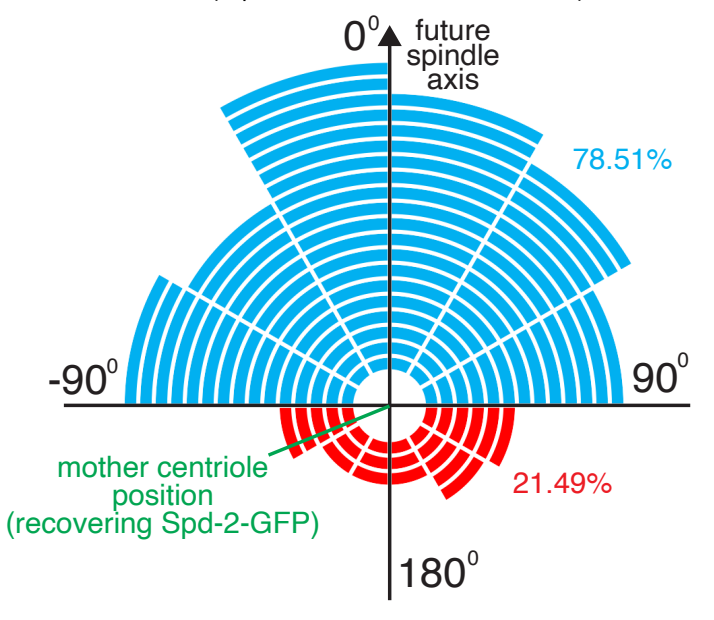

$\mathbf{F}$

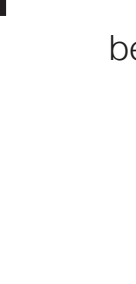

rose plot of angles between recovering RFP-Cnn position and recovering Spd-2-GFP position

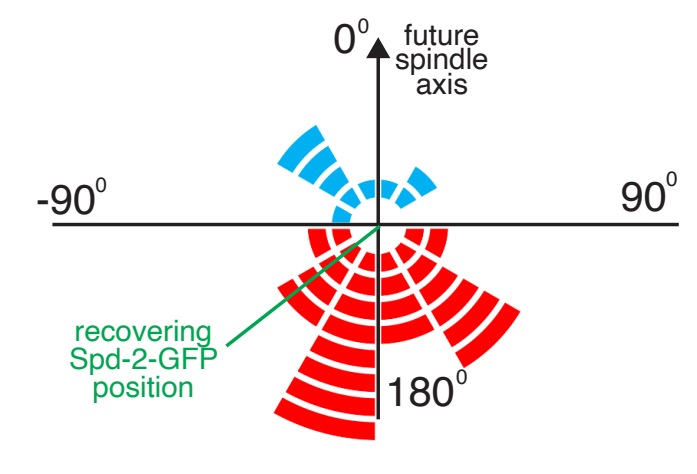

\section{G}

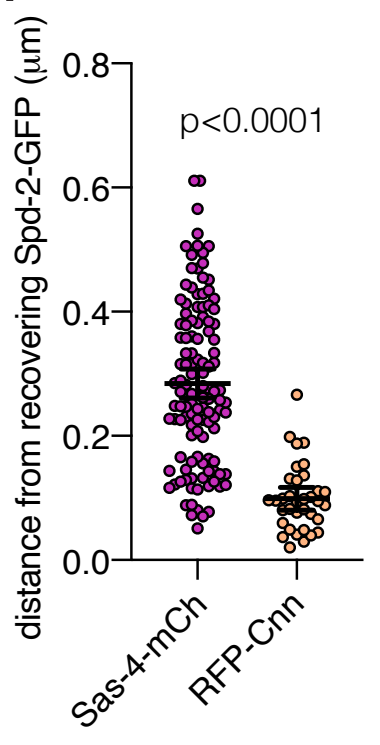




\section{Figure 2}

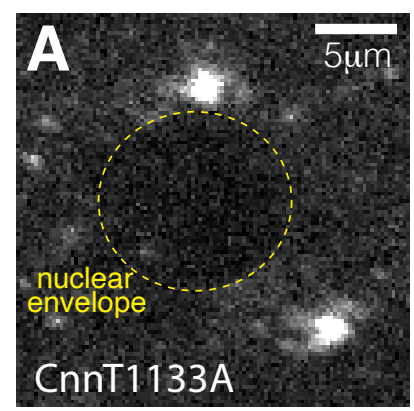

\section{attached centrosomes \\ C daughter centriole position \\ relative to mother centriole $(0,0)$ \\ and nuclear axis \\ (GFP-CnnT1133A;Sas-4-mCh)}

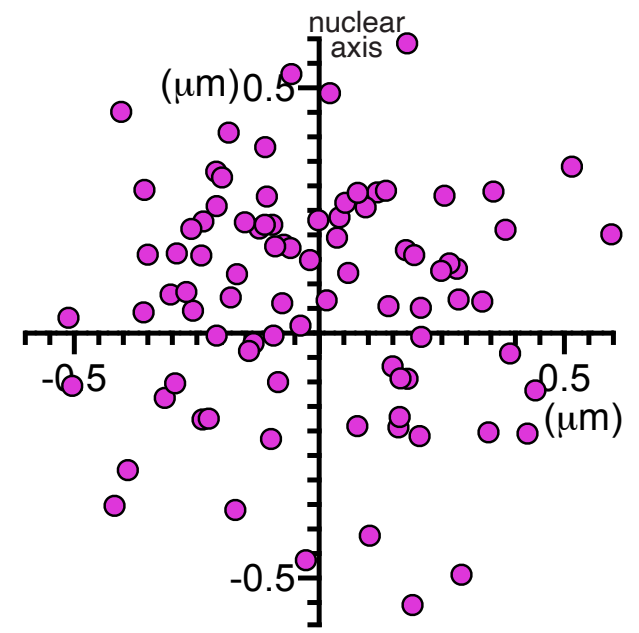

D Frequency distribution of daughter centriole angles relative to nuclear axis

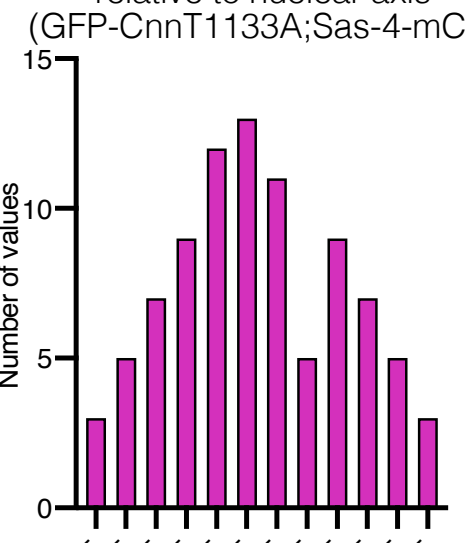

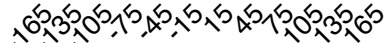

angle from centre of nucleus (bin center)

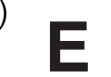

normal QQ plot for angles (GFP-CnnT1133A;Sas-4-mCh)

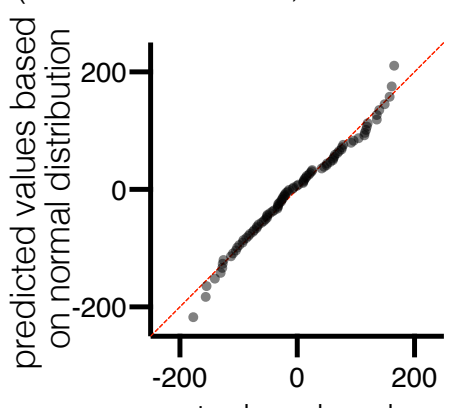
actual angle values

E rose plot for daughter centriole angle relative to nuclear axis

(GFP-CnnT1133A;Sas-4-mCh)

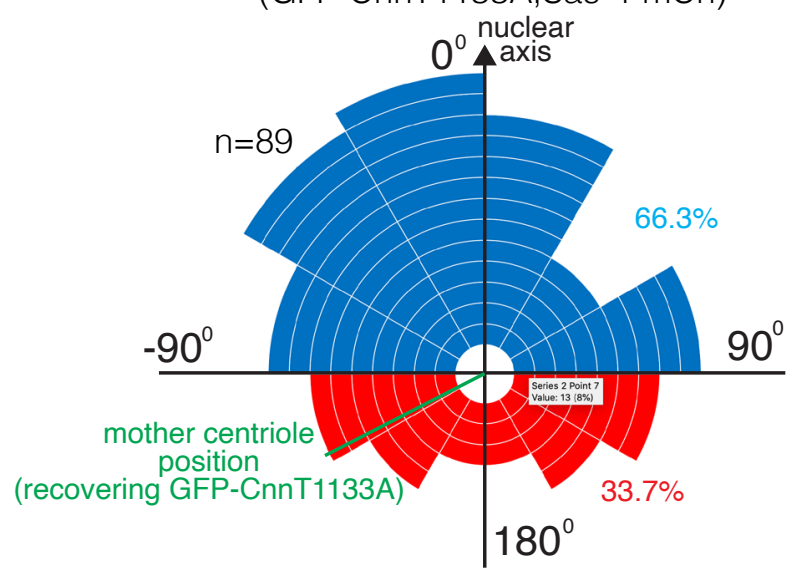

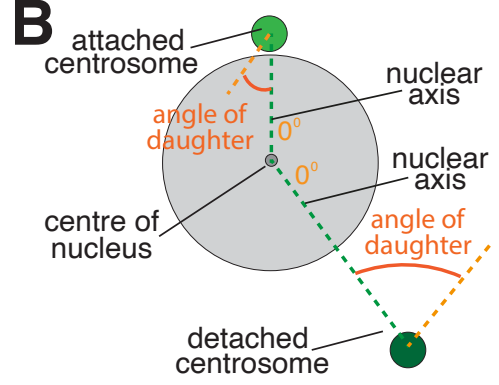

\section{detached centrosomes}

G daughter centriole position relative to mother centriole $(0,0)$ and nuclear axis (GFP-CnnT1133A;Sas-4-mCh)

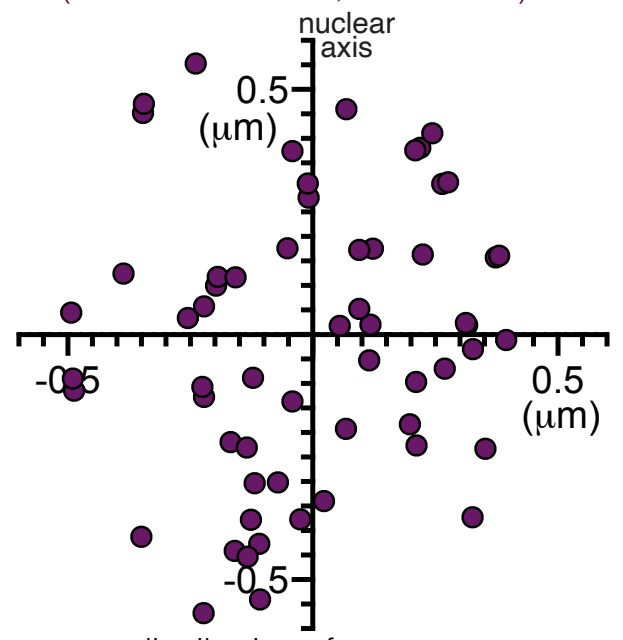

- Frequency distribution of daughter centriole angles relative to nuclear axis (GFP-CnnT1133A;Sas-4-mCh)

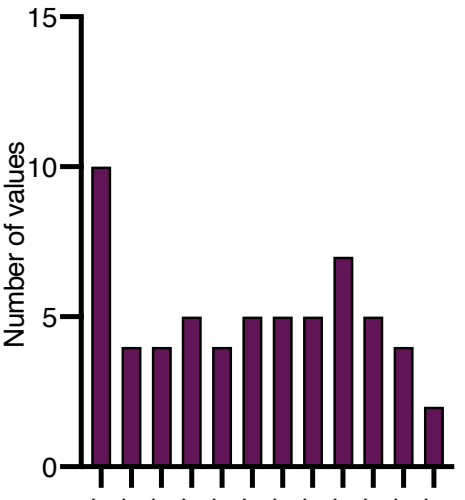
$60,3500,15,50,5,55015,05,30,60$ angle from centre of nucleus (bin center) rose plot for daughter centriole angle
relative to nuclear axis (GFP-CnnT1133A;Sas-4-mCh)

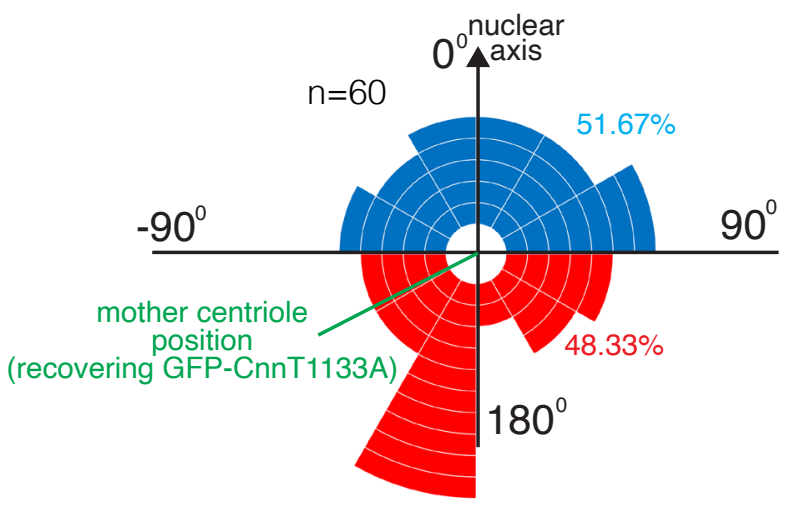

normal QQ plot for angles (GFP-CnnT1133A;Sas-4-mCh)

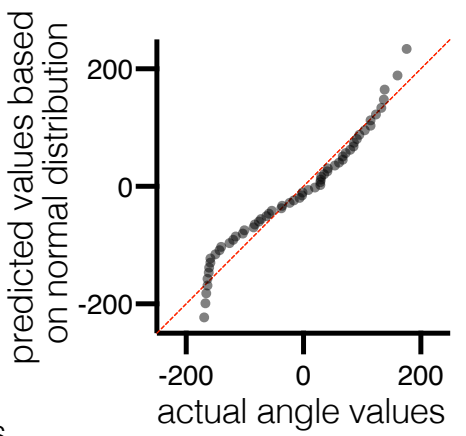

actual angle values

$$
\text { h) }
$$


Figure 3
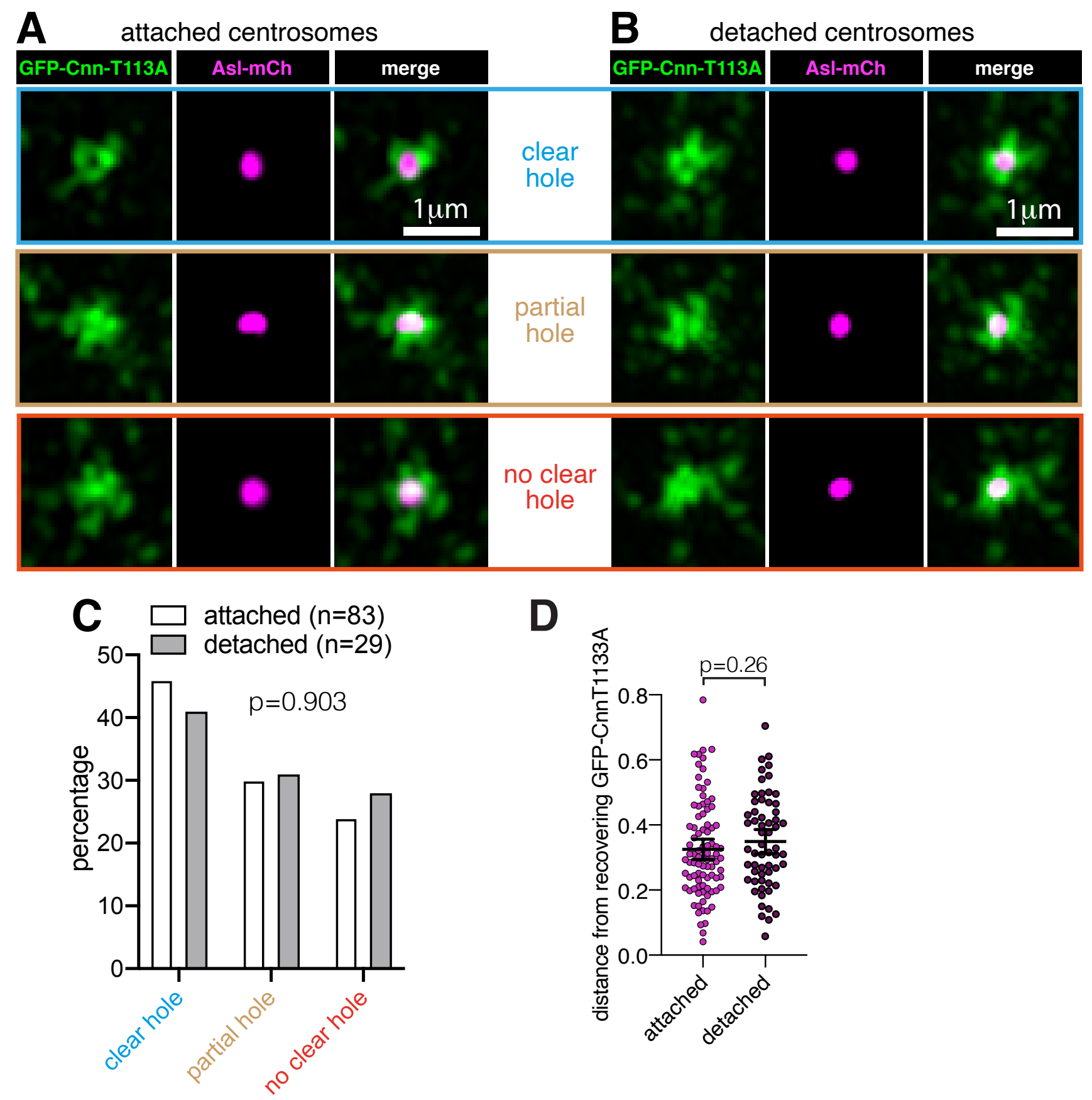
A
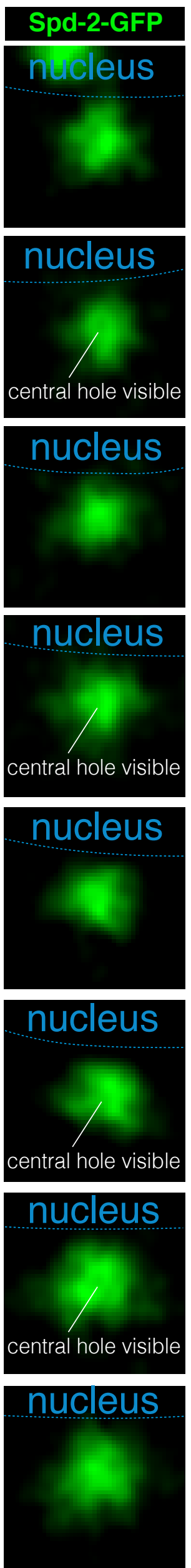

Figure 4 B
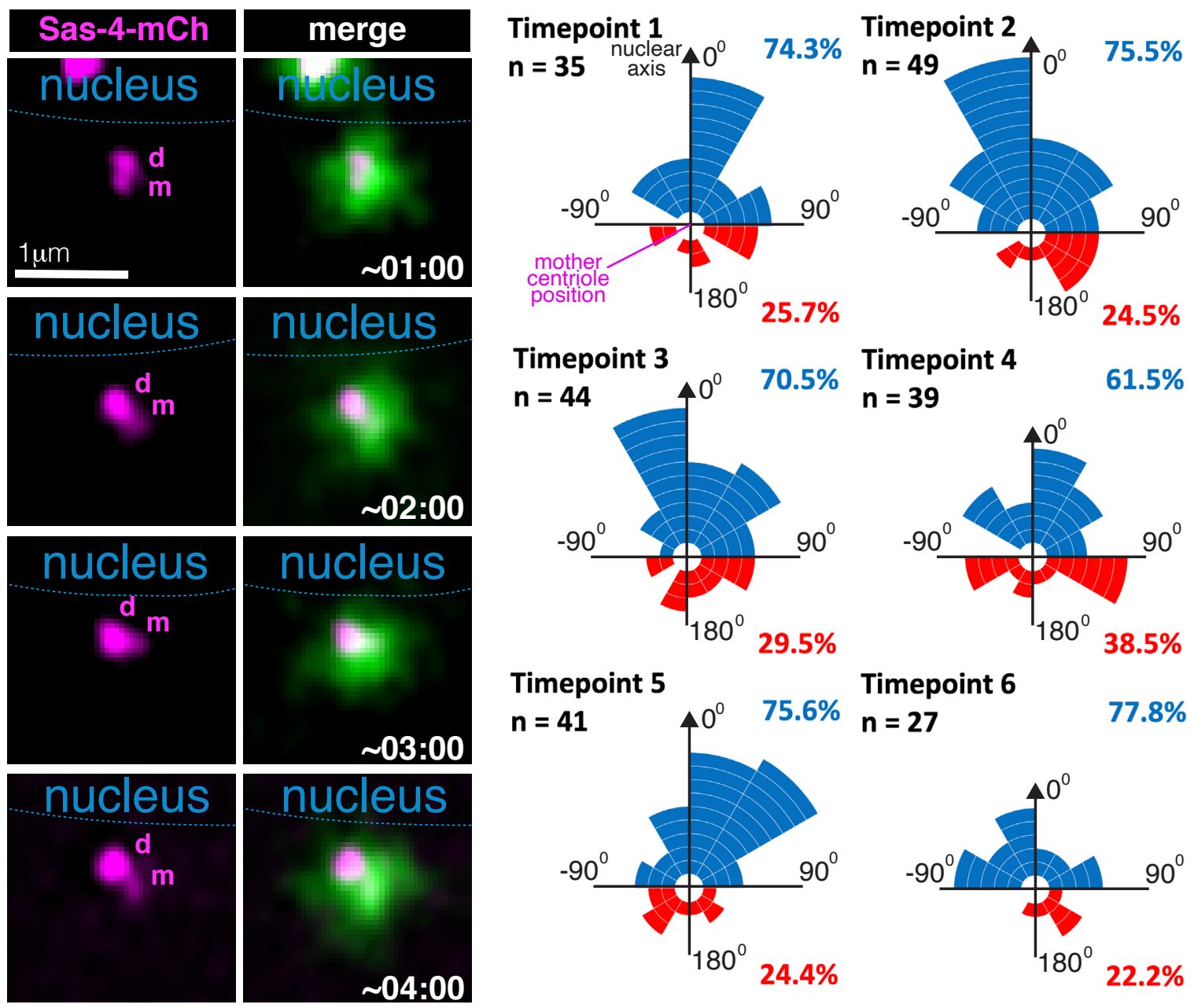

\section{Timepoint 3}

$\mathrm{n}=\mathbf{4 4}$

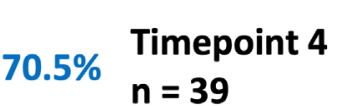

$24.5 \%$

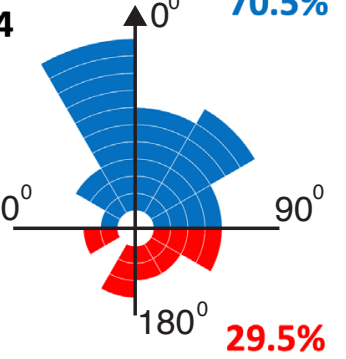

$=39$

$61.5 \%$

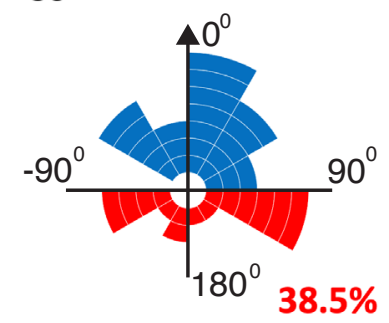

\section{Timepoint 5}

$\mathbf{n}=\mathbf{4 1} \quad \mathbf{4} 0^{0} \quad 75.6 \% \quad \mathbf{n}=\mathbf{2 7}$

$77.8 \%$
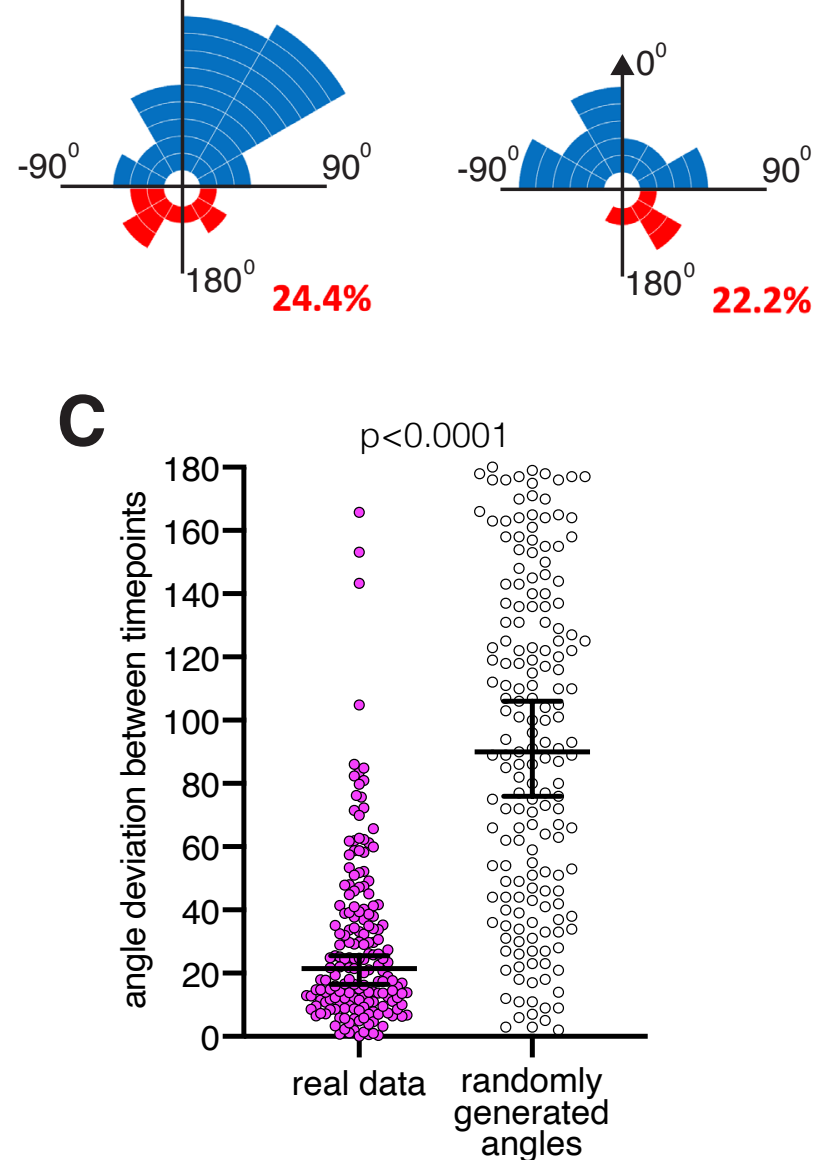

07:00

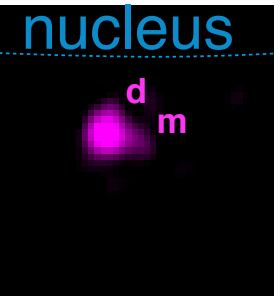




\section{Figure S1}

A

frequency distribution of daughter centriole angles

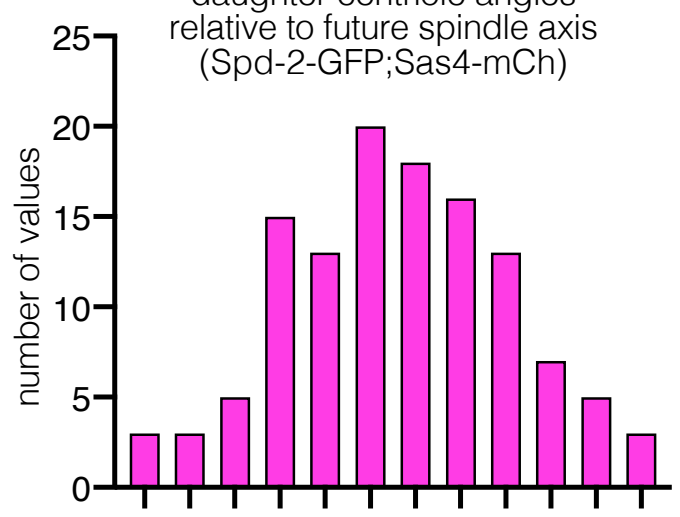

16

angle from future spindle axis (bin center)

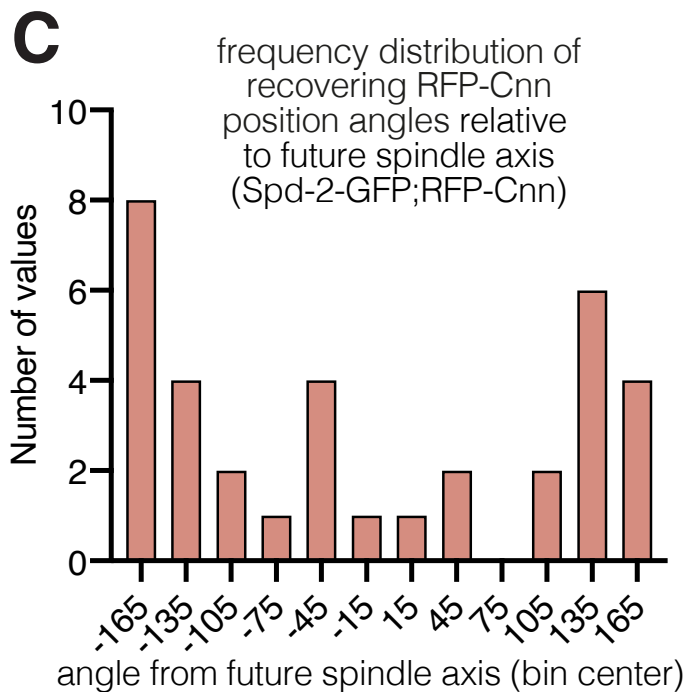

$B$

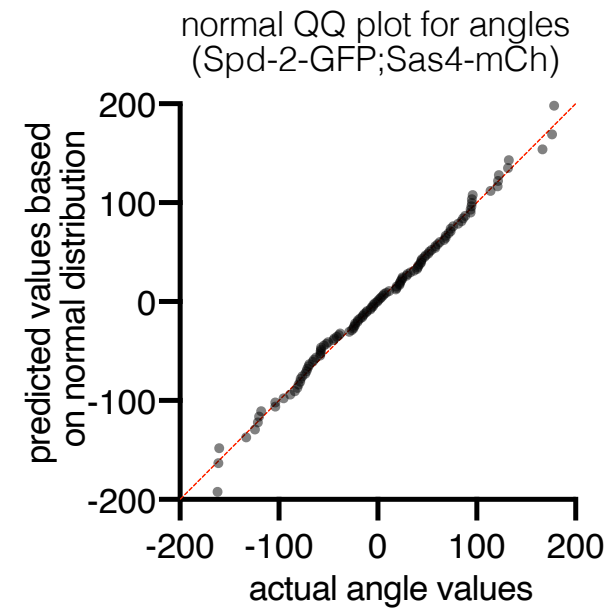

D

normal $Q Q$ plot for angles

(Spd-2-GFP;RFP-Cnn)

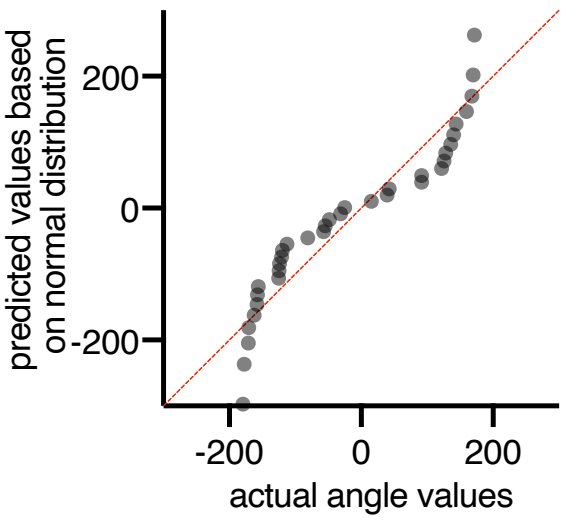

EE

daughter centriole position relative to mother centriole $(0,0)$ and future spindle axis

(Asl-mCh;Sas4-GFP)

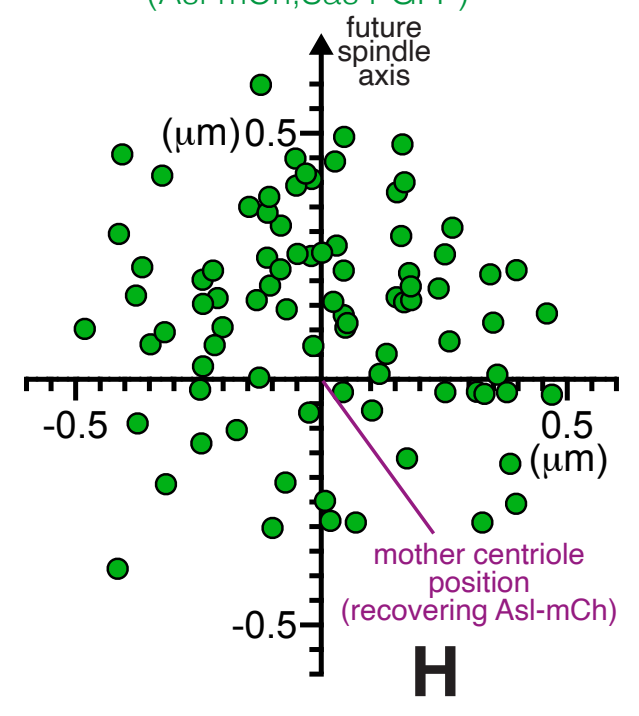

E
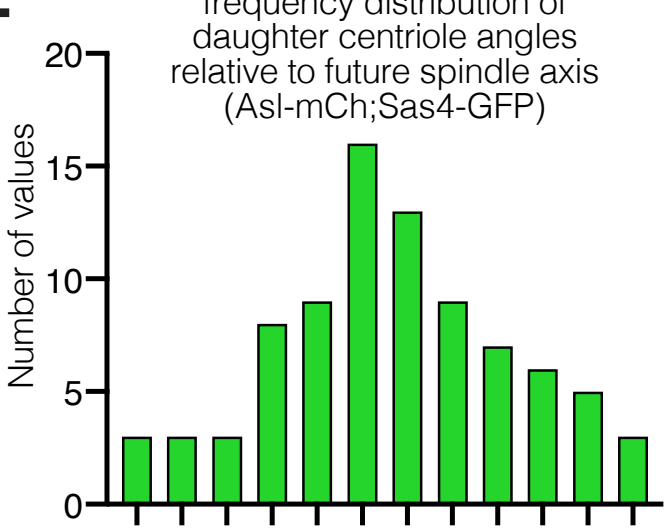

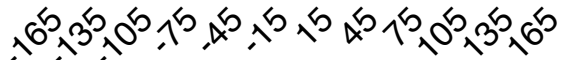
angle from future spindle axis (bin center)
G normal QQ plot for angles (Asl-mCh;Sas5GFP)

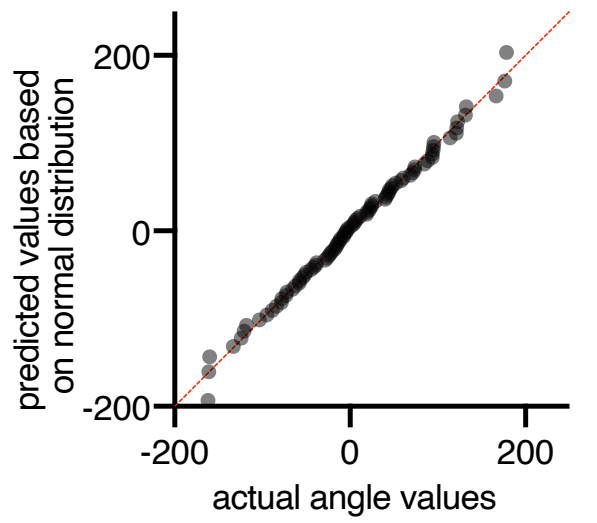




\section{Online Supplementary Material}

\section{Figure S1}

Further analysis of dual-colour FRAP data supports the finding that the site of daughter centriole assembly is non-random. (A) Frequency distribution of the angles at which daughter centrioles (marked by Sas-4-mCherry) form in relation to the future spindle axis $\left(0^{\circ}\right)$. (B) Normal $Q Q$ plot showing that the angles in $(A)$ conform well to a normal distribution. (C) Frequency distribution of the angles at which the recovering RFP-Cnn fluorescence is positioned in relation to the future spindle axis $\left(0^{\circ}\right)$. (D) Normal QQ plot showing that the angles in (B) do not conform well to a normal distribution. (E) Graph displays the estimated positions of daughter centrioles (green circles) relative to the estimated position of their respective mother centrioles (position 0,0 on the graph) and the future spindle axis (positive y-axis) obtained from AslmCherry (mother) Sas-4-GFP (daughter) data. (F) Frequency distribution of the angles at which daughter centrioles (marked by Sas-4-GFP) form in relation to the future spindle axis $\left(0^{\circ}\right)$. (G) Normal QQ plot showing that the angles in (F) conform well to a normal distribution. $(\mathbf{H})$ Rose plot representing the angle at which daughter centrioles (marked by Sas-4-GFP) form in relation to the future spindle axis $\left(0^{\circ}\right)$. Each segment corresponds to a single duplication event. Blue and red segments indicate daughter centriole assembly occurring less than or more than $90^{\circ}$ from the future spindle axis, respectively. (I) Graph showing the distance between the estimated positions of mother and daughter centrioles (left and right datasets) or two different estimations of the mother centriole (central dataset) in the different imaging conditions used, as indicated. Note that data for the datasets on the left and in the centre have been replotted from Figure $1 \mathrm{G}$ to allow comparison to the dataset on the right. Datasets were compared to each other using a one-way ANOVA Kruskal-Wallis test. 


\section{Figure S1}

A

frequency distribution of daughter centriole angles

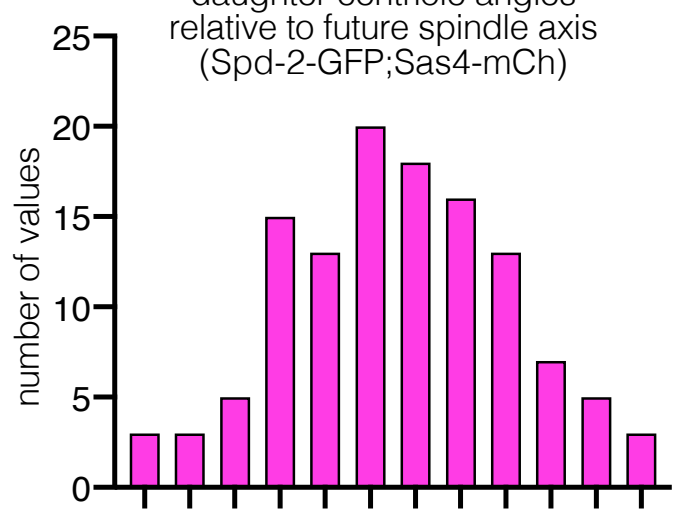

16

angle from future spindle axis (bin center)

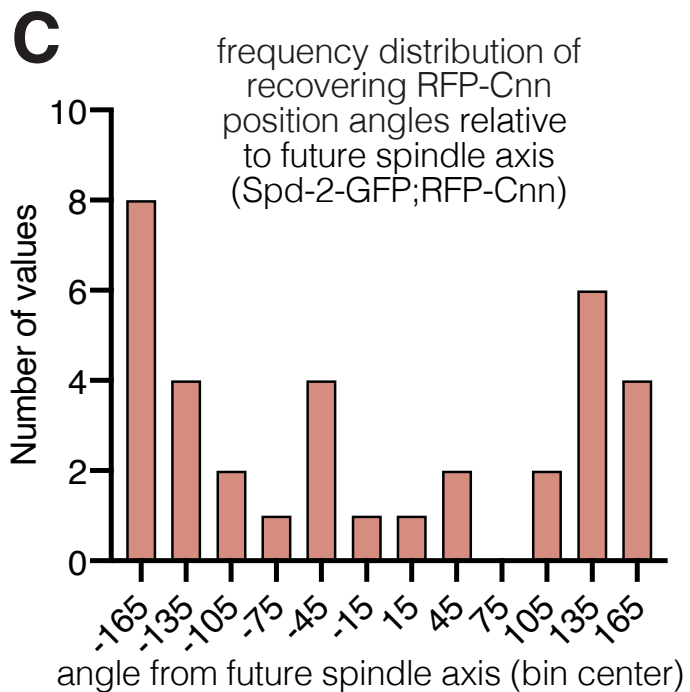

$B$

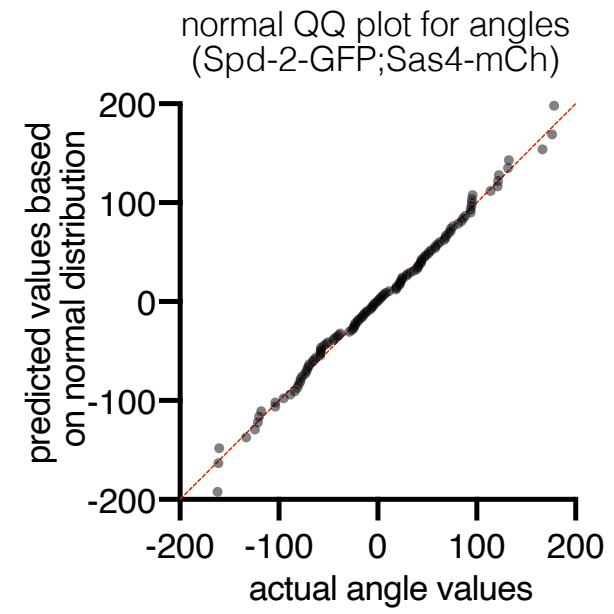

D

normal $Q Q$ plot for angles

(Spd-2-GFP;RFP-Cnn)

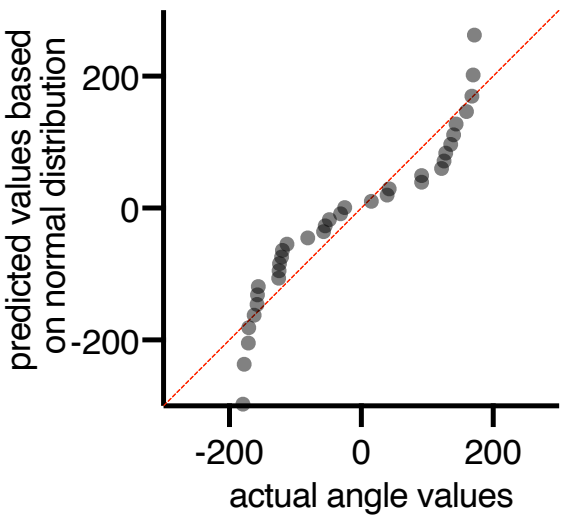

EE

daughter centriole position relative to mother centriole $(0,0)$ and future spindle axis

(Asl-mCh;Sas4-GFP)

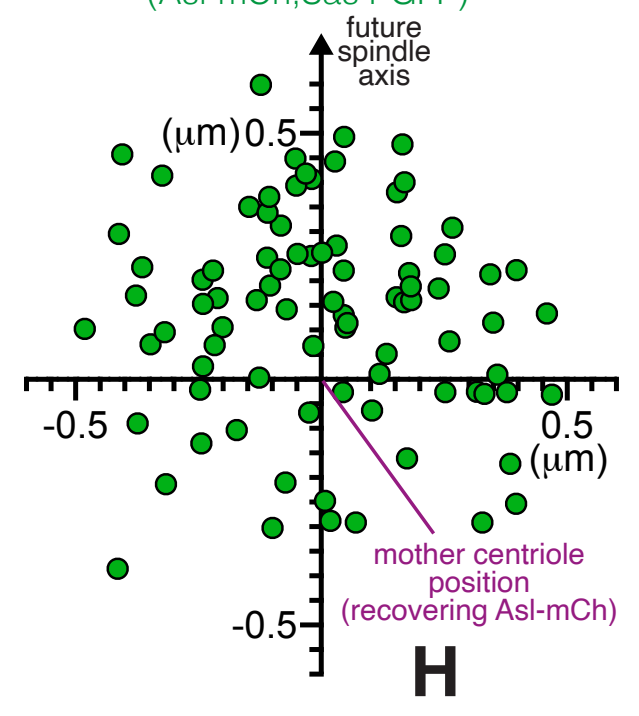

E
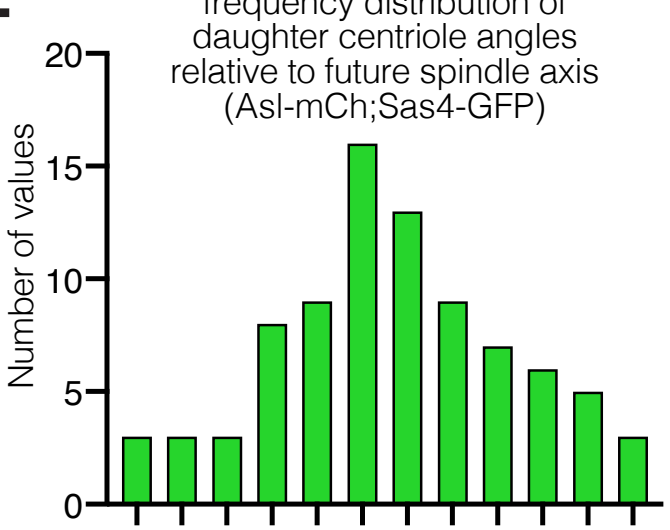

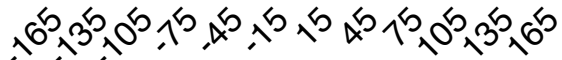
angle from future spindle axis (bin center)
G normal QQ plot for angles (Asl-mCh;Sas5GFP)

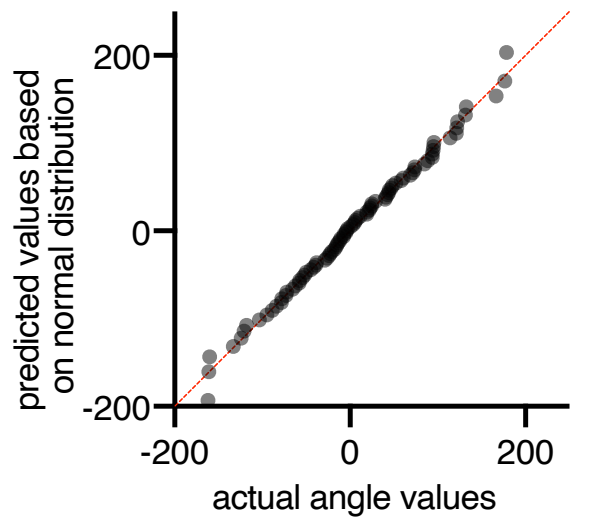

